\title{
La pêche côtière océanienne et son environnement
}

La dialectique instabilité/stabilité au fil de l'eau

\section{Gilbert David}

\section{(2) OpenEdition}

\section{Journals}

Édition électronique

URL : http://journals.openedition.org/jso/4352

DOI : $10.4000 /$ jso.4352

ISSN : $1760-7256$

Éditeur

Société des océanistes

Édition imprimée

Date de publication : 15 décembre 2008

Pagination : 247-270

ISBN : 978-2-85430-012-3

ISSN : 0300-953x

Référence électronique

Gilbert David, "La pêche côtière océanienne et son environnement », Journal de la Société des Océanistes [En ligne], 126-127 | Année 2008, mis en ligne le 15 décembre 2011, consulté le 17 juin 2020. URL : http://journals.openedition.org/jso/4352 ; DOI : https://doi.org/10.4000/jso.4352 


\section{La pêche côtière océanienne et son environnement : la dialectique instabilité/stabilité au fil de l'eau}

par

Gilbert DAVID*

\section{RÉSUMÉ}

De toutes les exploitations qui sont faites des ressources naturelles, la pêche est certainement l'une des plus vulnérables à l'instabilité du milieu naturel, que celle-ci concerne la météorologie (qui affecte la fréquence et la durée des sorties de pêche) ou les facteurs biotiques et abiotiques qui régissent la présence du poisson et son abondance sur les lieux de capture. Depuis des siècles, les pêcheurs océaniens ont su trouver des stratégies adaptatives face cette instabilité, qui se caractérisent par la diversité des biotopes exploités, le grand nombre des espèces cibles et la variété des moyens de production. Pour perdurer, l'activité halieutique doit être régulée. Les interdictions temporaires d'accès qui frappent une partie du territoire de pêche constituent le mode " traditionnel » de régulation. Face aux difficultés d'appliquer une gestion dite " moderne" des pêches, axée sur la collecte et le traitement de données statistiques concernant la ressource et la pression de pêche, un nombre grandissant de scientifiques proposent de mettre en auvre une gestion à données minima et de faire des aires marines protégées un nouvel outil de la gestion des ressources halieutiques, voire de revitaliser certaines règles ancestrales pour mette en place à l'échelle locale une co-gestion des ressources halieutiques.

MoTS-CLÉS : pêche vivrière, pêche artisanale, instabilité, vulnérabilité, adaptation, récifs, Océanie

De toutes les exploitations qui sont faites des ressources naturelles, la pêche est certainement l'une des plus vulnérables à l'instabilité des systèmes écologiques, que celle-ci affecte

\section{ABSTRACT}

The fishing activity is one of the most vulnerable natural resources uses with regard to natural environment instability. This instability depends essentially on a) the weather, which changes the frequency and the length of the fishing trips, $b$ ) the fish habitat and the sea nutrients contents which determine the fish populations abundance. To minimize their vulnerability to this instability, the Pacific islands fishermen have elaborated, through time, adaptative strategies based on the diversity of the fishing grounds, gears and target species. The traditional regulations are based on marine tenures and access control to the fishing grounds. Due to the difficulty of collecting fisheries statistics on a regular base in Pacific islands, an increasing number of scientists promote a) the participatory fish stock assessment, including data less management and $b$ ) the marine protected areas as new fish populations management tools.

KEYwORDS: village fisheries, instability, vulnerability, adaptation, reef ecosystem, Pacific islands

les ressources exploitées, la relation «prédateur proie » qui lie le pêcheur à ces dernières ou les relations entre l'océan et les ressources (figure 1). Le pêcheur doit composer avec la

* Unité S 140 Espace, IRD La Réunion, gilbert.david@la-reunion.ird.fr 


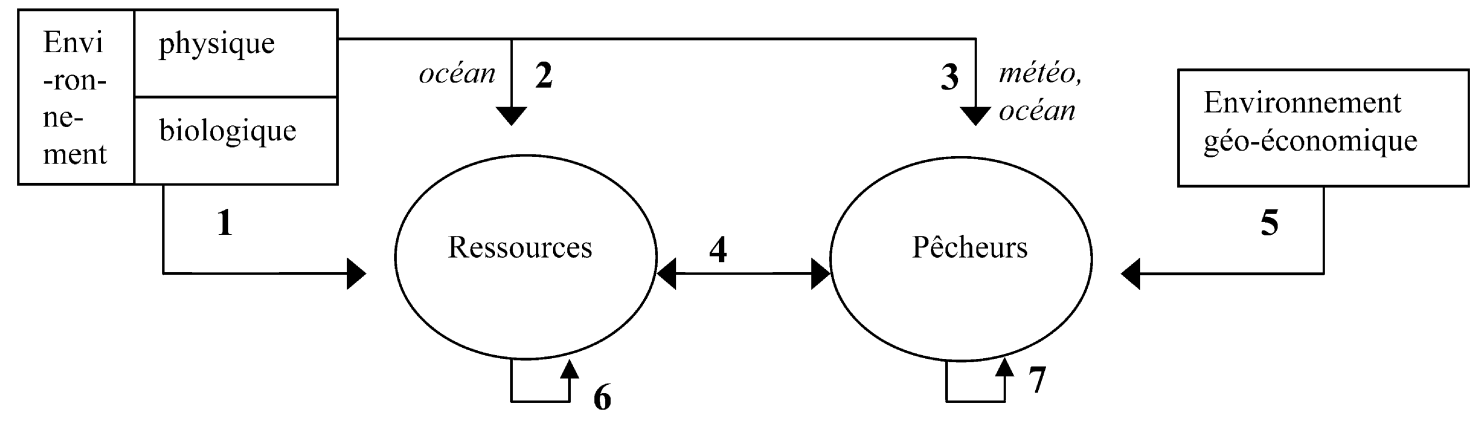

FIGURE 1. - Les sept formes d'instabilité affectant la pêche

nature; plus son équipement est simple et peu coûteux ${ }^{1}$ plus sa vulnérabilité aux aléas naturels croît.

L'Océanie insulaire ${ }^{2}$ offre un bel exemple de cette vulnérabilité. La pêche y est l'une des activités les moins utilisatrices de capitaux financiers qui soient au monde mais elle concerne des dizaines de milliers de personnes, une large part de la population littorale pratiquant une activité halieutique (Dalzell et al., 1996). La compréhension de cette vulnérabilité et des régulations adaptatives que les pêcheurs d'Océanie ont mis en place au fil des siècles constitue l'objet du présent article. Il s'appuie sur une recherche effectuée de 1983 à 1991 sur la pêche villageoise de Vanuatu (David, 1991, Cillaurren et David, 1995, Cillaurren et al., 2001), complétée de réflexions plus récentes portant sur la notion de risque (David, 2004) et de travaux en cours sur la résilience (David, 2005). Il se structure en six points. Dans le premier sera dressé un bref panorama de la pêche côtière océanienne. Seront ensuite présentés les aléas naturels affectant l'activité halieutique, le cas spécifique du milieu corallien étant évoqué lors d'un troisième point. Le quatrième point portera sur la réduction des instabilités affectant la pêche. Seront successivement passées en revue: l'adaptation des pêcheurs aux aléas naturels puis la régulation «traditionnelle» de l'activité halieutique. Le cinquième point sera consacré à la gestion moderne de la pêche artisanale pour finir sur une présentation des aires marines protégées comme nouvel outil de gestion des ressources halieutiques.

\section{La pêche côtière océanienne : présentation générale}

La pêche côtière océanienne, que nous appellerons également pêche villageoise dans la suite du texte (tableau 1) se divise en deux ensembles - la pêche vivrière et la pêche artisanale dont les dynamiques spatiotemporelles induisent des dialectiques instabilité/stabilité très différentes.

Par pêche vivrière, on entend l'activité halieutique dont plus de la moitié de la production est destinée à la consommation du pêcheur et de sa famille. Il en existe deux types : la pêche vivrière d'autosubsistance qui ne fait l'objet d'aucune commercialisation et la pêche vivrière commerciale, dont au maximum $49 \%$ de la production est mise sur le marché. Ces ventes sont essentiellement de proximité et portent sur les excédents par rapport à l'autoconsommation des familles de pêcheurs. Ces deux types de pêche s'exercent sur les zones intertidales et infratidales de moins de 20 mètres de profondeur composées des platiers et lagons coralliens, de la partie supérieure des pentes récifales et des écosystèmes associés : mangroves, herbiers de phanérogames, plages, embouchures de rivières. Environ 4000 espèces de poissons coralliens ont été relevées en Océanie insulaire (Meyers, 1989). Une large part d'entre elles est pêchée à des fins alimentaires. À cette fin, un large éventail d'engins, actifs (sagaies, arcs, éperviers, fusils sous-marins, sabre d'abattis) comme passifs (casiers, filets maillants, lignes, parcs à poisson) est utilisé (Anell, 1955; Bataille-Benguigui, 1981). La plupart d'entre

1. On considérera qu'une partie du capital de recherche et développement inclus dans tout moyen de production du secteur technique sert à réduire la vulnérabilité humaine vis-à-vis des contraintes naturelles.

2. Par Océanie insulaire, on entend l'ensemble des terres émergées de l'Océanie moins le continent australien et les deux grandes îles que sont la Nouvelle-Guinée $\left(785000 \mathrm{~km}^{2}\right)$ et la Nouvelle Zélande $\left(268680 \mathrm{~km}^{2}\right)$. 
eux appartiennent au pêcheur ou au ménage auquel il se rattache, les pêches collectives devenant de plus en plus rares. Les sorties se font à pied, en plongée sous-marine ou à l'aide de pirogues monoxyles de 2 à 5 mètres de long, généralement propulsées à la pagaie. Autrefois très communes, les pirogues à voile ne sont plus guère utilisées, hormis dans certaines zones de Mélanésie. La motorisation des pirogues progresse sous l'impulsion de programmes gouvernementaux - ainsi, depuis vingt ans, des pirogues moto- risées en fibres synthétiques et sans balancier, ou en contreplaqué avec balancier, se multiplientelles parmi les pêcheurs des Salomon et de Kiribati.

Ces pêcheries assurent encore l'essentiel de la production halieutique villageoise sur l'ensemble des vingt-deux pays et territoires relevant de la Communauté Pacifique (tableau 1), toutefois l'ichtyosarchotoxisme ${ }^{3}$ qui sévit dans de nombreuses zones constitue fréquemment un frein à la consommation.

TABlEAU 1. - Structure de la production halieutique villageoise et production par habitant (d'après SPC, 1993 ; Dalzell et Adams, 1994)

\begin{tabular}{|c|c|}
\hline Pays ou territoires & $\begin{array}{l}\text { Pêche } \\
\text { vivrière } \\
\text { ( \% pêche } \\
\text { totale) }\end{array}$ \\
\hline Mélanésie & 78 \\
\hline Fiji & 71 \\
\hline Nouvelle-Calédonie & 66 \\
\hline Papouasie Nlle-Guinée & 81 \\
\hline Salomon & 90 \\
\hline Vanuatu & 81 \\
\hline Micronésie & 77 \\
\hline États fédérés & 91 \\
\hline Guam & 81 \\
\hline Kiribati & 74 \\
\hline Marshall & 84 \\
\hline Nauru & 26 \\
\hline Mariannes du Nord & 63 \\
\hline Palau & 50 \\
\hline Polynésie & 68 \\
\hline Samoa américaines & 81 \\
\hline Cook & 87 \\
\hline Wallis-Futuna & 86 \\
\hline Niue & 90 \\
\hline Pitcairn & 100 \\
\hline Polynésie française & 52 \\
\hline Tokelau & 100 \\
\hline Tonga & 40 \\
\hline Tuvalu & 87 \\
\hline Samoa occidentales & 94 \\
\hline Région CPS & 76 \\
\hline $\begin{array}{l}\text { Région CPS } \\
\text { (Papouasie Nlle-Guinée } \\
\text { exceptée) }\end{array}$ & 75 \\
\hline
\end{tabular}

Total
production
halieutique
$(\mathrm{t})$

64954

22706

3032

25554

11150

2512

24353

6889

586

12324

2369

377

322

1486

15351

267

982

1000

115

8

5999

191

2362

927

3500

104658

79104

Pêche
vivrière/
Populat.
du pays
(kg/hab.an)

$$
9,4
$$

21,8

11,5

5,2

30,5

13,5

42,4

55,9

3,4

124

41,5

10,3

4, 1

48,1

18,8

4,4

49,3

62

46,8

160

15,4

119

9,6

88,7

20,4

12,6

24,9

$\begin{array}{cc}\begin{array}{c}\text { Pêche } \\ \text { commer-ciale/ }\end{array} & \begin{array}{c}\text { Production } \\ \text { totale/ }\end{array} \\ \text { Popdu pays } & \text { Pop. du pays } \\ \text { (kg/hab.an) } & \text { (kg/hab.an) }\end{array}$

$\begin{array}{cc}2,6 & 12 \\ 8,8 & 30,6 \\ 5,9 & 17,4 \\ 1,2 & 6,4 \\ 3,5 & 34 \\ 3,0 & 16,5 \\ 12,4 & 54,8 \\ 5,8 & 61,7 \\ 0,8 & 4,2 \\ 44 & 168 \\ 7,5 & 49 \\ 29 & 39,3 \\ 2,4 & 6,5 \\ 47,2 & 95,3 \\ 9 & 27,8 \\ 1,1 & 5,5 \\ 7,1 & 56,4 \\ 9,9 & 71,9 \\ 5,5 & 52,3 \\ 0 & 160 \\ 14,4 & 29,8 \\ 0 & 119 \\ 14,7 & 24,3 \\ 13,2 & 101,9 \\ 1,3 & 21,7 \\ 3,9 & 16,5 \\ 8,2 & 33,1\end{array}$

(Papouasie Nlle-Guinée

25

3. Communément appelé « ciguatéra », l'ichtyosarchotoxisme est provoqué par une toxine (la ciguatoxine), élaborée par un dinoflagellé (microalgue unicellulaire) benthique, Gambierdiscus toxicus, épiphyte d'algues macroscopiques peuplant les récifs coralliens, notamment les formes ramifiées ou touffues (Taylor, 1985). L'ingestion de G. toxicus par les poissons brouteurs de corail entraîne un empoisonnement de leur chair et de leurs organes qui se transmet à leurs prédateurs. Par bioaccumulation dans la chaîne alimentaire, les toxines initialement produites par la microalgue se concentrent pour atteindre chez certaines espèces de poissons des taux susceptibles d'intoxiquer les consommateurs humains, le risque augmentant avec la taille et l'âge du poisson ingéré. Chez l'homme, la ciguatoxine agit principalement sur le système nerveux en modifiant la perméabilité cellulaire aux ions sodium, ce qui entraîne une dépolarisation de la fibre nerveuse qui se traduit par des symptômes digestifs, neurologiques, cardio-vasculaires et un état général de grande faiblesse. Dans les cas extrêmes, heureusement rares, l'ichtyosarchotoxisme provoque des paralysies, le coma et parfois la mort (Laurent et al., 1993). 
La pêche artisanale se focalise soit sur les ressources démersales profondes (100 à $500 \mathrm{~m}$ ) des pentes récifales externes, soit sur les ressources pélagiques (thonidés et daurades coryphènes, notamment) qui s'agrégent autour des dispositifs de concentration de poissons (DCP) mouillés sur des fonds de 500 à $2000 \mathrm{~m}$ à moins de dix milles des côtes (Cillaurren, 1999; Desurmont et Chapman, 2000). Dans un cas comme dans l'autre, la pêche se fait à l'aide d'embarcations de 5 à $11 \mathrm{~m}$ propulsées par un moteur hors-bord, équipées de palangrottes ou de lignes de traînes montées sur des moulinets à main (Cillaurren et al. 2001). Exceptés les « Poti-marara » de Polynésie française, bateaux très rapides destinés à l'origine à pêcher les grands pélagiques au harpon (Blanchet et al., 1987; Blanchet et Borel, 1988), cette flottille est souvent de piètre qualité nautique. Son uniformité, comme celle des espèces cibles (les ressources pélagiques agrégées par des DCP et les poissons démersaux profonds), s'explique par le rôle prépondérant joué par la Communauté Pacifique (CPS) ${ }^{4}$ dans l'émergence de cette pêche. Les logiques mises en œuvre relèvent de la rationalité halieutique et économique (Reveret, 1991). Elles visent à accroître la production halieutique tout en veillant à ne pas dépasser le niveau optimal d'exploitation, matérialisé par la prise maximale équilibrée (PME), cette dernière ayant été estimée à partir des pêches expérimentales effectuées par la CPS (Dalzell et Preston, 1992). Comme pour toute pêche artisanale, l'essentiel de la production est destinée à la commercialisation. Les circuits de distribution vont parfois jusqu'aux capitales et principaux centres urbains des pays concernés, le transport se faisant par route et voie aérienne. Notons que les ressources démersales profondes, composées de lutjanidés (vivaneaux et perches), de serranidés (mérous), de léthrinidés (bossus) et de pentapodidés (brèmes), présentent l'avantage d'être exempts d'ichtyosarchotoxisme, ce qui facilite leur commercialisation.

\section{Caractérisation de l'instabilité : les aléas naturels affectant l'activité halieutique}

L'activité halieutique sur les lieux de pêche est régie par deux corollaires. Il s'agit en premier lieu de la présence du poisson et de son abondance, en second lieu de la présence du pêcheur ${ }^{5}$.

\section{Les facteurs limitant l'accès des pêcheurs sur les lieux de capture}

En dehors de toute considération sociale, la présence du pêcheur sur les lieux de capture est sous la dépendance de facteurs météorologiques, principalement la fréquence et l'intensité des pluies ainsi que la vitesse et la direction des vents, et de facteurs hydrologiques qui, induits par les premiers, façonnent la morphologie de la surface de l'océan et déterminent l'état de la mer. Ce sont d'une part l'amplitude, la longueur d'onde, la célérité et la direction de propagation des vagues générées par le vent dans la zone de pêche (figure 2), d'autre part la direction de propagation de la houle, son amplitude et sa période, cette dernière déterminant la célérité et la longueur d'onde de la houle (Guilcher, 1979). Tous ces facteurs déterminent les conditions de travail sur les lieux de pêche et affectent la décision du pêcheur de s'y rendre ou de différer sa sortie. Quand les vents forcissent jusqu'à devenir des tempêtes puis des cyclones, c'est l'ensemble des constructions et des infrastructures de transport, de conservation et de transformation de la production halieutique ainsi que la flotte de pêche qui peuvent être menacés.

La plupart des facteurs météorologiques et hydrologiques affectant l'activité halieutique peuvent être amplifiés ou réduits par la configuration du littoral ou la morphologie de l'arrière pays. Plus le pêcheur se rapproche de la terre, plus la variabilité de ces facteurs augmente. À cet égard, la question se pose en termes différents pour la zone intertidale et les lieux de pêche situés au-delà, qui ne sont accessibles que par voie de mer. Hormis la ligne des brisants, la zone intertidale est beaucoup moins affectée par l'état de la mer. Les lieux les plus exposés se situent sur les plages et rivages rocheux soumis aux houles ou aux vents dominants. Les moins exposés correspondent aux embouchures de rivières, fréquemment protégées par des flèches de sable ou de galets, aux plages de fond de baie, ainsi qu'aux mangroves et aux herbiers de phanérogames qui ne se développent que sur des sédiments fins, se déposant à l'abri des houles du large et des vagues engendrées par les vents dominants. Dans ces sites abrités, ce sont beaucoup plus les conditions atmosphériques, notamment la pluie et le froid, qui commandent la fréquentation des lieux de pêche que l'état de la mer.

4. La Communauté Pacifique est la principale organisation régionale d'appui technique au développement. Jusqu'à la fin des années 1990, elle s'est appelée " Commission du Pacifique Sud».

5. On considèrera que le pêcheur exerce sur un stock un prélèvement dont les effets sont déterminés par l'effort de pêche qu'il a déployé et sa productivité, assimilée à la production du pêcheur par unité de temps. Cette productivité dépend à la fois de l'abondance de la ressource et de l'efficacité de l'engin employé, c'est-à-dire le rapport entre le nombre d'animaux présents sur l'aire de pêche et les captures. 


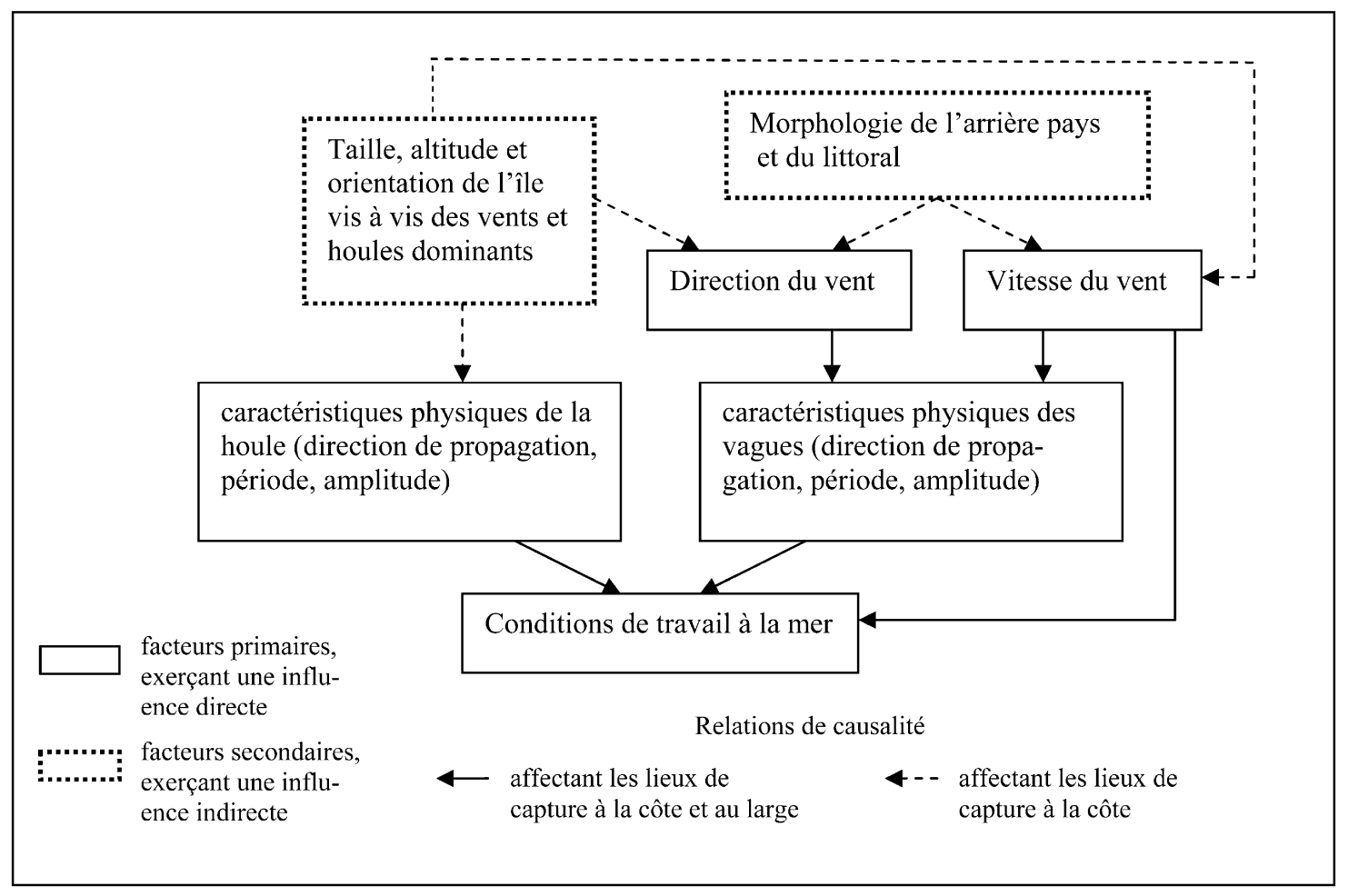

FIGURE 2. - Facteurs abiotiques déterminant la présence ou l'absence des pêcheurs sur les lieux de capture

Vanuatu est, à ma connaissance, l'unique pays $\mathrm{du}$ Pacifique pour lequel une étude détaillée concernant les effets du climat sur l'activité halieutique a été conduite (David, 1991). Ceux-ci varient d'abord selon la latitude. Ainsi à un sud ventilé par les alizés de secteur sud-est, où la mer est souvent grosse et se prête difficilement au travail sur de petites embarcations, peut-on opposer un nord moins exposé à l'alizé où l'influence du climat équatorial se traduit par un pourcentage élevé de calmes et de vents de faible intensité, surtout en période estivale, durant laquelle les conditions de travail à la mer sont particulièrement clémentes. Cette partie septentrionale de l'archipel est nettement plus pluvieuse que le sud et donc moins propice à la mise en œuvre de procédés simples et peu coûteux de conservation du poisson que sont le séchage ou l'association salage-séchage, qui tous deux requièrent une bonne ventilation et l'humidité relative la plus faible possible. Si on considère l'exposition à l'aléa cyclonique comme le principal facteur présidant au choix d'un site où implanter une infrastructure très coûteuse comme une unité de transformation du poisson ou un centre de construction navale, il convient de privilégier le nord du pays, où les cyclones et dépressions tropicales sont moins fréquents (figure 3).
À l'échelle de chaque île, l'exposition par rapport au vent dominant constitue un second type de variation. Exposées à l'alizé et battues par les houles océanes, les façades orientales et sud-est des îles se révèlent moins propices à la pêche que les côtes sous le vent, qui bénéficient de leur situation abritée et d'une moindre pluviosité, plus favorable à la conservation du poisson fumé, salé ou séché. Ainsi dans la partie nordouest de l'archipel, une vaste zone abritée des houles du sud-est épouse la forme d'un $\mathrm{V}$ dont Epi constituerait la pointe, les îles Malakula et Santo la branche occidentale, la branche orientale se composant d'Ambrym, de Pentecôte et de Maéwo.

\section{Les facteurs favorisant ou limitant l'abondance du poisson}

L'absence ou la présence et l'abondance des poissons sur les lieux de pêche dépendent principalement de la manière dont les facteurs environnementaux assurent les fonctions d'abri, d'équilibre physiologique et de nutrition des espèces marines (figure 4).

Les facteurs abiotiques permettant à ces dernières de s'abriter relèvent de la géologie, de la morphologie et de la sédimentologie sous- 


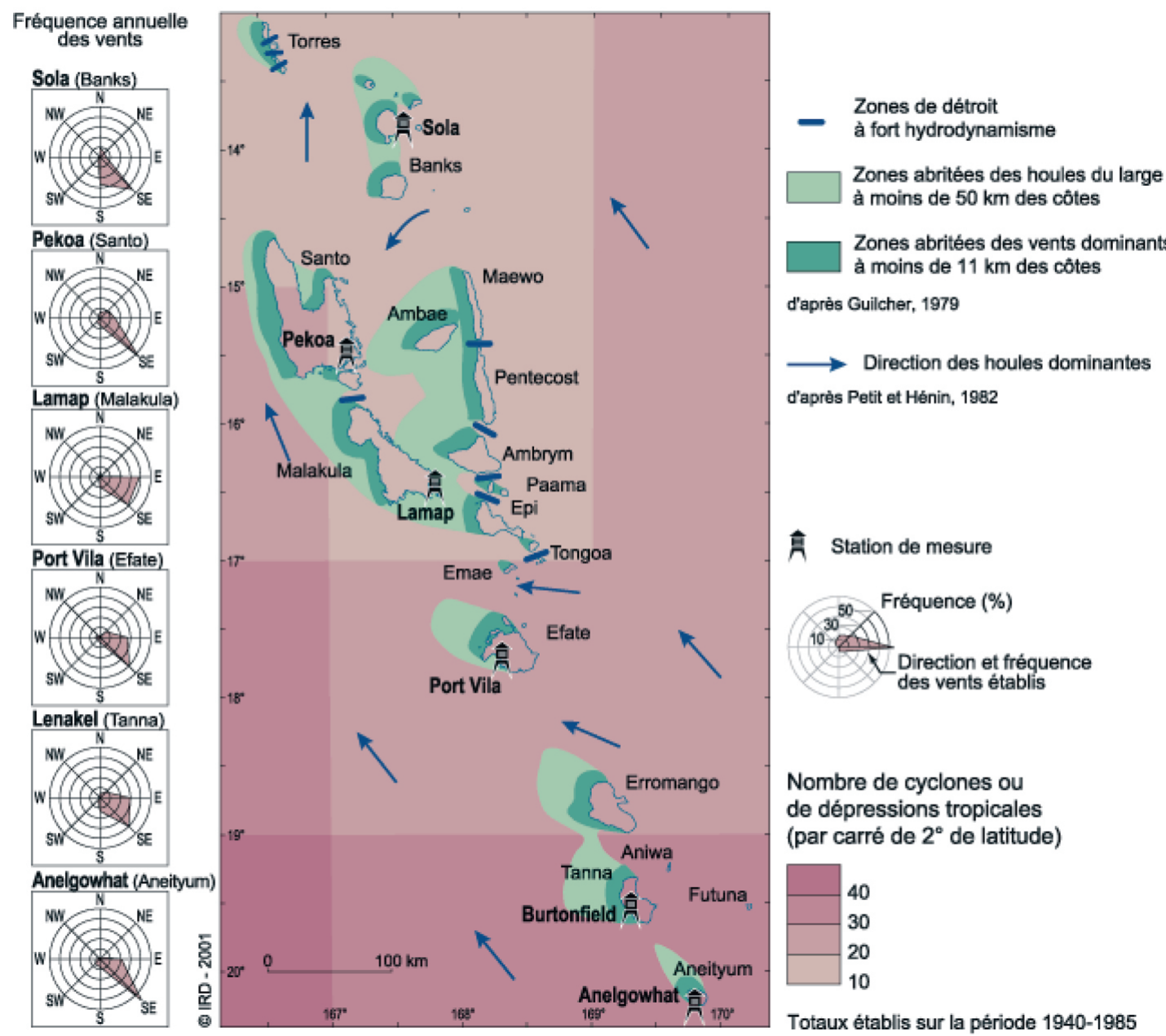

FIGURE 3. - Le climat de Vanuatu et ses implications sur la pêche (Cillaurren et al. 2001)

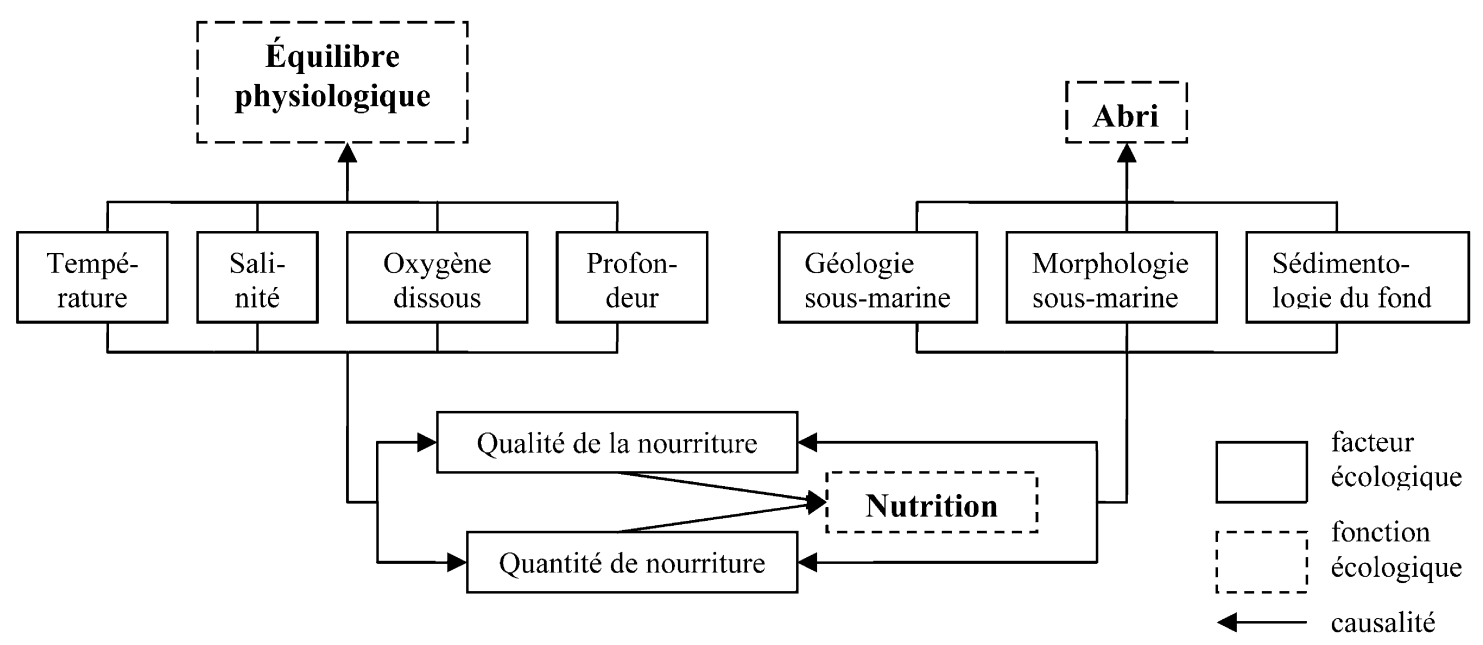

FIGURE 4. - Typologie des facteurs contrôlant l'abondance des poissons sur les lieux de pêche selon les fonctions écologiques qu'ils assurent 


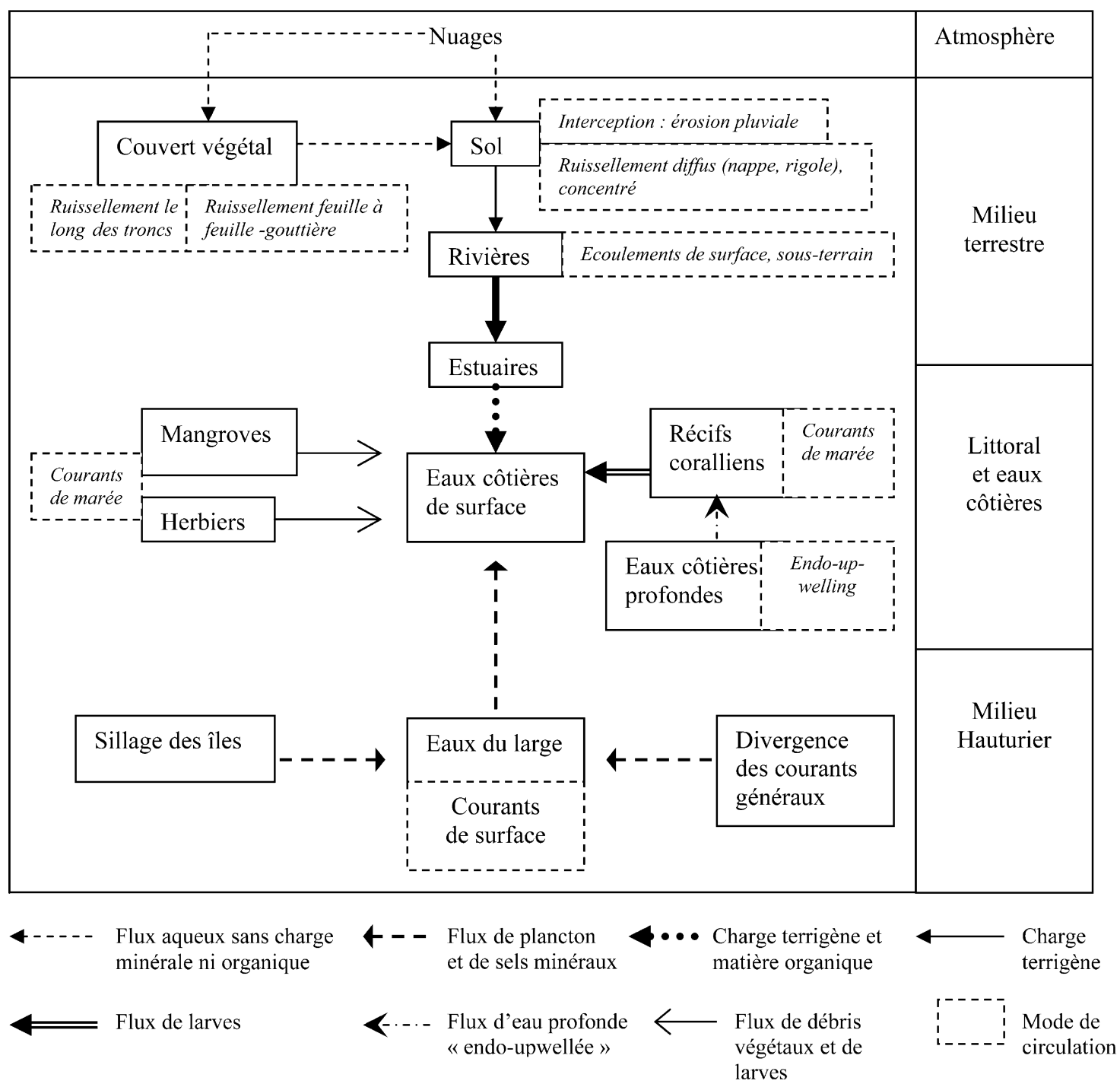

FIGURE 5. - Schéma de circulation des flux de matériel nutritif approvisionnant les eaux côtières

marine. Quant aux facteurs d'ordre physicochimiques favorisant ou perturbant l'équilibre physiologique du poisson, ils sont au nombre de quatre : la température, la salinité, la profondeur et la teneur en oxygène. La fonction de nutrition se décline selon deux variables : la qualité et la quantité de nourriture disponible pour chaque poisson composant la population exploitée. Des trois fonctions écologiques (abri, équilibre physiologique, nutrition) déterminant la présence ou l'absence de poissons dans les lieux de pêche, la dernière est celle qui présente le réseau le plus complexe d'interrelations entre facteurs écologiques (figure 5), réseau dont il convient de préciser maintenant les principales composantes.

À toute étape de la vie d'un poisson et selon l'espèce à laquelle il appartient correspondent des besoins physiologiques particuliers, qui déterminent ses déplacements à des fins alimentaires ou de reproduction. La quantité et la qualité de nourriture en un lieu dépendent de la concentration des eaux en matériel nutritif et de la composition de celui-ci en organismes vivants, en sels minéraux ou en matières organiques mortes, sous formes dissoute ou particulaire.

En eau côtière, le matériel nutritif présent sur les lieux de pêche a trois origines : hauturière, terrestre et littorale. Le matériel nutritif d'origine hauturière résulte de quatre processus distincts : a) le déplacement des courants généraux; b) l'apparition en saison chaude au voisinage des îles ou en plein océan de densités très élevées de cyanobactéries fixatrices d'azote du genre Trichodesmium, qui forment de vastes tourbillons océaniques dont le tracé évolue au gré des turbulences affectant les courants marins 
qui circulent entre des îles rapprochées jusqu'à former des «sillages d'îles" (Dupouy et al., 1988) ; c) des remontées d'eau profonde, dont la concentration en sels nutritifs est nettement plus élevée que celle des eaux de surface. Ce phénomène se manifeste au voisinage direct des îles dont les fonds sont très pentus et peut être assimilé à un " upwelling localisé »; d) les remontées par capillarité d'eau profonde à travers les structures coralliennes, phénomène appelé endoupwelling ", pourraient également jouer un rôle significatif dans l'enrichissement des eaux côtières (Rougerie et Wauthy, 1986).

Le matériel nutritif d'origine terrestre provient soit du lessivage des versants en bord de mer, soit de l'érosion des sols des îles hautes aux versants pentus, dont il est fréquent que les sommets soient abondamment arrosés par les précipitations. Dans ce contexte, toute forte pluie d'une durée minimale d'une heure ${ }^{6}$ produit un ruissellement diffus, puis concentré, chargé en particules terrigènes qui va alimenter le réseau hydrographique. Arrivant en mer, ce flux hydrique va générer un panache de turbidité qui va venir enrichir ou perturber le milieu côtier, notamment les herbiers de phanérogames marines, les mangroves et les récifs coralliens.

Le matériel nutritif d'origine littorale est émis sous les formes de flux de larves ou de débris végétaux plus ou moins décomposés par les herbiers de phanérogames marines, les mangroves et les récifs coralliens qui assurent également une fonction d'abri pour nombre de poissons côtiers.

\section{L'instabilité inhérente à l'espace de pêche : le cas du milieu corallien}

En raison de l'absence de plateau continental, les archipels de l'Océanie insulaire ne disposent que d'un espace de pêche côtier extrêmement réduit. Dans de nombreuses îles d'origine volcanique, les fonds sous-marins sont extrêmement pentus et il n'est pas rare qu'à un kilomètre de la ligne de rivage des profondeurs inférieures à $500 \mathrm{~m}$ soient atteintes. Dans ce contexte géologique si particulier, le milieu corallien forme le principal espace de pêche exploité par la pêche villageoise. Il se compose de cinq ensembles principaux : a) la zone à éperons et sillons de la pente externe, b) le tombant de la pente interne, c) le platier récifal, d) le lagon, e) les passes, également appelés hoa selon leur dénomination polynésienne lorsqu'elles séparent deux îlots coralliens, appelés motus. Les deux premiers espaces sont disposés parallèlement à la ligne de rivage ; le dernier l'est perpendiculairement : il est alors transverse aux deux espaces précédents, qu'il met en communication. D'un strict point de vue hydrologique, la zone à éperons et sillons de la pente externe peut être assimilée à un espace " filtre », puisqu'elle marque le contact entre les récifs frangeants ou barrières et la houle du large dont elle tend à dissiper une petite partie de l'énergie en freinant sa progression au voisinage $\mathrm{du}$ fond. La passe, quant à elle, peut être considérée comme un espace corridor, facilitant la propagation des flux de marée entre un espace émetteur et un espace récepteur, alternativement la zone à éperons et sillons de la pente externe ou le tombant de la pente interne selon le rythme de la marée (figure 6).

À cette diversité du paysage corallien répond une extrême diversité spécifique des peuplements ichtyologiques : il n'est pas rare que près d'une centaine d'espèces de poissons soit répertoriée sur un seul hectare de récifs. Ainsi en 1988, lors $\mathrm{du}$ recensement des ressources marines de Vanuatu, un total de 469 espèces de poissons a été identifié de manière visuelle (Williams, 1990). $\mathrm{Au}$ total, les récifs abriteraient environ un quart des espèces connues de poissons marins (Moberg and Rönnback, 2003). Les contraintes pesant sur la présence et l'abondance des poissons sont essentiellement d'ordre écologique. La grande diversité spécifique qui caractérise l'ensemble des écosystèmes de la zone intertidale et du tombant récifal sur lesquels s'exerce l'effort de pêche traditionnel s'accompagne d'une forte spécialisation des espèces, tant du point de vue alimentaire que de l'habitat, et d'une forte dispersion géographique des poissons d'une même espèce (Kulbicki, 1992). Les biotopes favorables à chaque espèce n'occupent que des espaces de dimension réduite - on parlera de microbiotopes - généralement discontinus les uns par rapport aux autres. L'ensemble de ces microbiotopes forme dans l'espace une sorte de mosaïque à trois dimensions dont chaque élément abrite une micropopulation composée d'un petit nombre de poissons d'une même espèce. En raison de leur éloignement géographique à l'échelle du territoire de pêche, les migrations entre deux micropopulations de la même espèce sont souvent réduites. Sur une courte période de temps, quelques semaines à un mois, chaque micropopulation peut donc être considérée comme un isolat évoluant selon une dynamique démographique qui lui est propre. À chacune de ces micro-populations correspond donc un micro-

6. L'action érosive d'une pluie dont la taille des gouttes dépasse celle du crachin commence pour une durée de précipitation minimale de $30 \mathrm{mn}$ (Batti, 2005). 


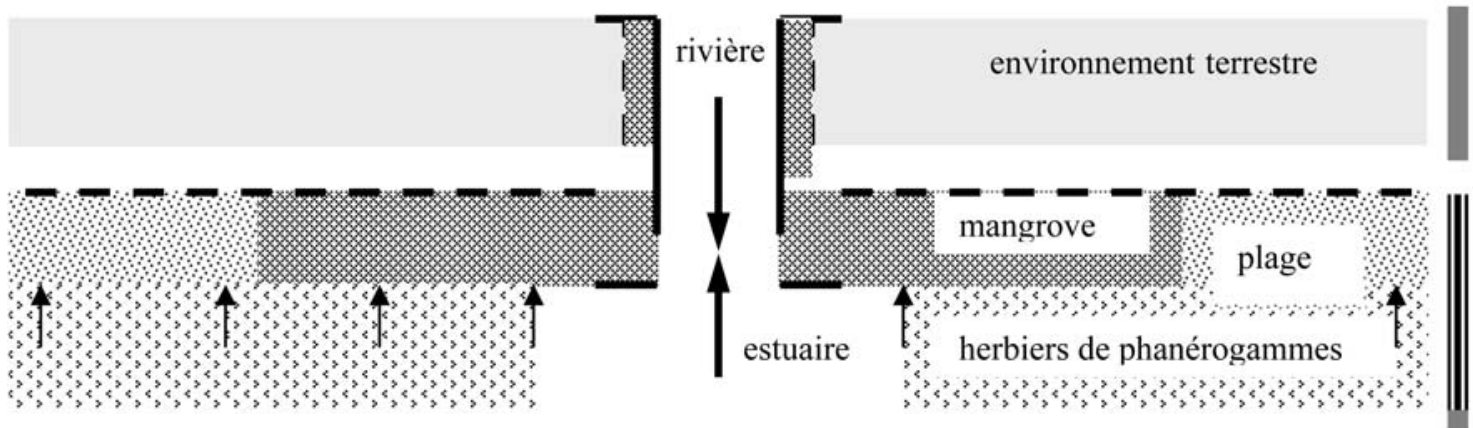

Lagon

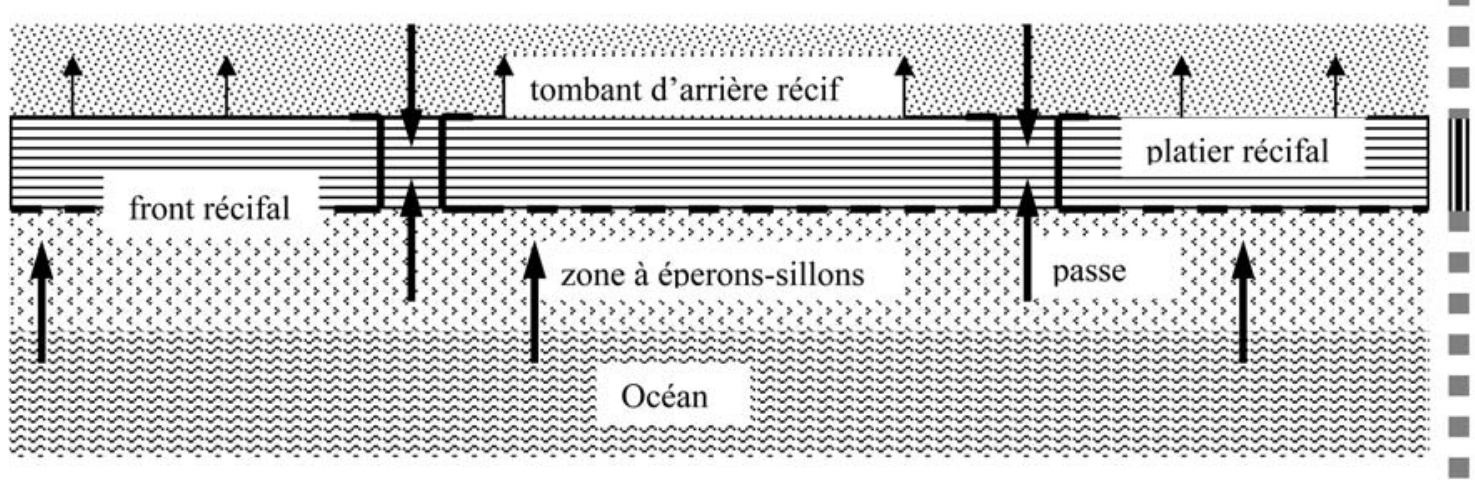

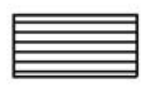

Espace filtre de grande efficacité Espace filtre de moindre efficacité

Espace rétrolittoral

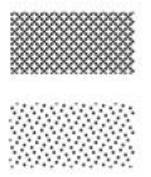

Espace filtre et récepteur

Espace récepteur

Zone intertidale
† Flux de faible énergie

4 Flux de forte énergie Espace $\quad-\quad-$ Espace corridor - - barrière

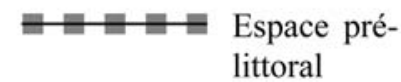

FigURE 6 - Schéma des structures et fonctions des littoraux de l'Indo-Pacifique en termes de flux d'énergie

stock, composé de la fraction de la micropopulation qui peut être capturée par les engins de capture mis en œuvre par les pêcheurs.

Compte tenu de leur taille réduite, ces microstocks sont sensibles à toute exploitation intensive de la part des pêcheurs et leur abondance à l'hectare est faible. Ces deux facteurs, conjugués à la grande diversité spécifique de la biomasse de poissons, constituent des contraintes essentielles pour l'activité halieutique. S'y ajoutent les variations saisonnières de l'abondance des poissons, particulièrement marquées dans le cas des petits pélagiques, "maquereaux et sardines" (Grandperrin et al, 1982 ; Conand, 1987). Ces contraintes sont rédhibitoires pour le pêcheur, il ne peut les contourner et doit obligatoirement les intégrer à ses stratégies et techniques de captures.

\section{La réduction de l'instabilité}

\section{L'adaptation des pêcheurs aux aléas naturels affectant leur activité}

La diversité des espèces cibles et celle des biotopes exploités constituent les principales adaptations de la pêche traditionnelle aux contraintes affectant la ressource. La première s'appuie sur la grande diversité spécifique du stock et apporte une réponse logique d'une part à la faible abondance des micro-stocks unités monospécifiques qui le composent, et d'autre part aux variations saisonnières affectant ces stocks. Ainsi lorsqu'une ou plusieurs espèces se raréfient, que ce soit en raison de causes naturelles, comme un mauvais recrutement ${ }^{7}$, ou d'une trop grande 
exploitation, il est possible de reporter l'effort de pêche sur les autres espèces qui demeurent présentes en abondance sur les lieux de pêche. L'analyse de 943 sorties de pêche réalisées fin 1984 sur l'ensemble de l'archipel du Vanuatu a montré que plus de 100 espèces de poissons, appartenant à 32 familles, étaient couramment capturées par la petite pêche villageoise ${ }^{8}$.

D'un point de vue halieutique, les passes constituent un espace privilégié où les poissons, passant de l'océan au lagon et vice versa, se concentrent. La méthode la plus efficace pour les capturer est d'installer au débouché de la passe, côté lagon, des parcs à poissons, formés de plusieurs dizaines de mètres d'un grillage de type poulailler, d'environ $1,50 \mathrm{~m}$ de haut, tendu sur des pieux de fer ou de bois fichés dans le corail. Cette technique est particulièrement prisée en Polynésie (Blanchet et al., 1985), où des parcs à poissons sont également mis en place dans la partie supérieure du tombant de la pente interne (Echinard, 1972). La partie sommitale de la pente externe est, en revanche, trop battue pour les y installer; dans ce milieu, la pêche se pratique à la ligne à main ou en plongée sous-marine lorsque la mer est calme. Au-delà, l'accès aux zones de pêche nécessite l'emploi d'une embarcation. C'est le domaine de la pêche à la ligne, que celle-ci soit mise en œuvre en surface (pêche à la traîne) ou au fond (palangrottes), et de la chasse sous-marine qui se pratique dans la première vingtaine de mètres et se révèle particulièrement intéressante pour la capture des langoustes et des coquillages nacriers, trocas et burgaux, qui forment la principale ressource économique de cet espace (Bour, 1990).

Cette diversité des biotopes exploités est chose commune pour la plupart des pêcheurs artisanaux du monde entier lorsque l'activité halieutique se pratique à l'échelle de l'année. En revanche, il est beaucoup plus rare qu'au cours d'une même sortie, un pêcheur prospecte à la fois le platier récifal, les premiers mètres du tombant, et un autre biotope : plage, mangrove ou embouchure de rivière, comme on l'observe à Vanuatu. Changer de biotope constitue une excellente parade aux aléas météorologiques. Lorsque la mer est mauvaise et interdit toute sortie en pirogue, il est toujours possible de se réfugier dans les embouchures de rivières, dans les baies abritées ou dans les mangroves et d'y redéployer l'effort de pêche sur une ou plusieurs espèces cibles selon l'abondance de la ressource et sa diversité spécifique (figure 7).

La diversité des moyens de production est la conséquence logique de cette diversité des espèces cibles et des biotopes exploités par la pêche vivrière. Chaque type d'engins n'exerce en effet son effort de pêche que sur un nombre réduit d'espèces. La vulnérabilité d'une espèce de poissons à un engin de pêche dépend à la fois a) de la morphologie du poisson, notamment de sa forme, de sa taille et de la grandeur de sa bouche $^{9}$, b) de son comportement alimentaire ${ }^{10}$, c) de la structure de son habitat (plage, crevasses coralliennes, patates de corail, ...) et de la profondeur de cet habitat ${ }^{11}$. Limiter l'effort de pêche à un seul engin aurait donc pour conséquence de se priver d'un grand nombre d'espèces comestibles non capturables par l'engin en question mais accessibles à d'autres engins. Ce souci de s'adapter aux variations d'abondance et de vulnérabilité des espèces exploitées conduit les pêcheurs à posséder plusieurs types d'engins et surtout à en utiliser plus d'un au cours d'une même sortie, ce qui est beaucoup plus remarquable. Lors des 943 sorties de pêche mentionnées plus haut, dix types d'engins ont été identifiés. Sept d'entre eux ont été utilisés soit seuls, soit en association avec un ou plusieurs autres types; les trois autres ont toujours été utilisés en associations. Au total 39 types d'engins ou associations d'engins ont été répertoriés (22 associations de deux types d'engins, 9 associations de 3 types d'engins, une association de 4 types d'engins et 7 utilisations d'un seul engin).

Cette diversité des moyens de production n'est pas uniquement la conséquence directe de la diversité des espèces cibles, elle est également induite par le souci qu'ont les pêcheurs d'exploiter les biotopes les plus divers de manière à minimiser les risques de non-pêche que font peser sur eux les conditions météorologiques et l'état de la mer. D'une part, certains types d'engins de pêche sont mieux adaptés à certains biotopes que d'autres. D'autre part, selon les conditions changeantes du milieu, il peut s'avérer précieux de pouvoir choisir entre plusieurs types d'engins

8. Fruit d'une collaboration étroite entre le Département de la statistique de Vanuatu et l'Orstom, cette enquête s'insérait dans le premier recensement halieutique qui ait été conduit dans ce pays (David, 1985, 1987, 1988, 1991). Ses résultats n'ont jamais fait l'objet d'une publication spécifique.

9. Ainsi n'est-il guère efficace de pêcher la sardine à l'aide d'un fusil sous-marin, la taille du poisson est trop petite, un filet serait nettement plus approprié.

10. Il est inutile d'espérer capturer un poisson herbivore avec un hameçon appâté d'un mollusque ou pêcher de jour une espèce qui ne « sort » que la nuit.

11. Ainsi tendre en surface un filet droit pour capturer des espèces démersales vivant à une dizaine de mètres de profondeur n'est-il guère réaliste, la chasse sous-marine ou la ligne serait certainement plus efficace. 


\section{Contraintes affectant la présence des pêcheurs sur les lieux de pêche}

Conditions météorologiques et état de la mer

\section{Contraintes affectant la ressource}
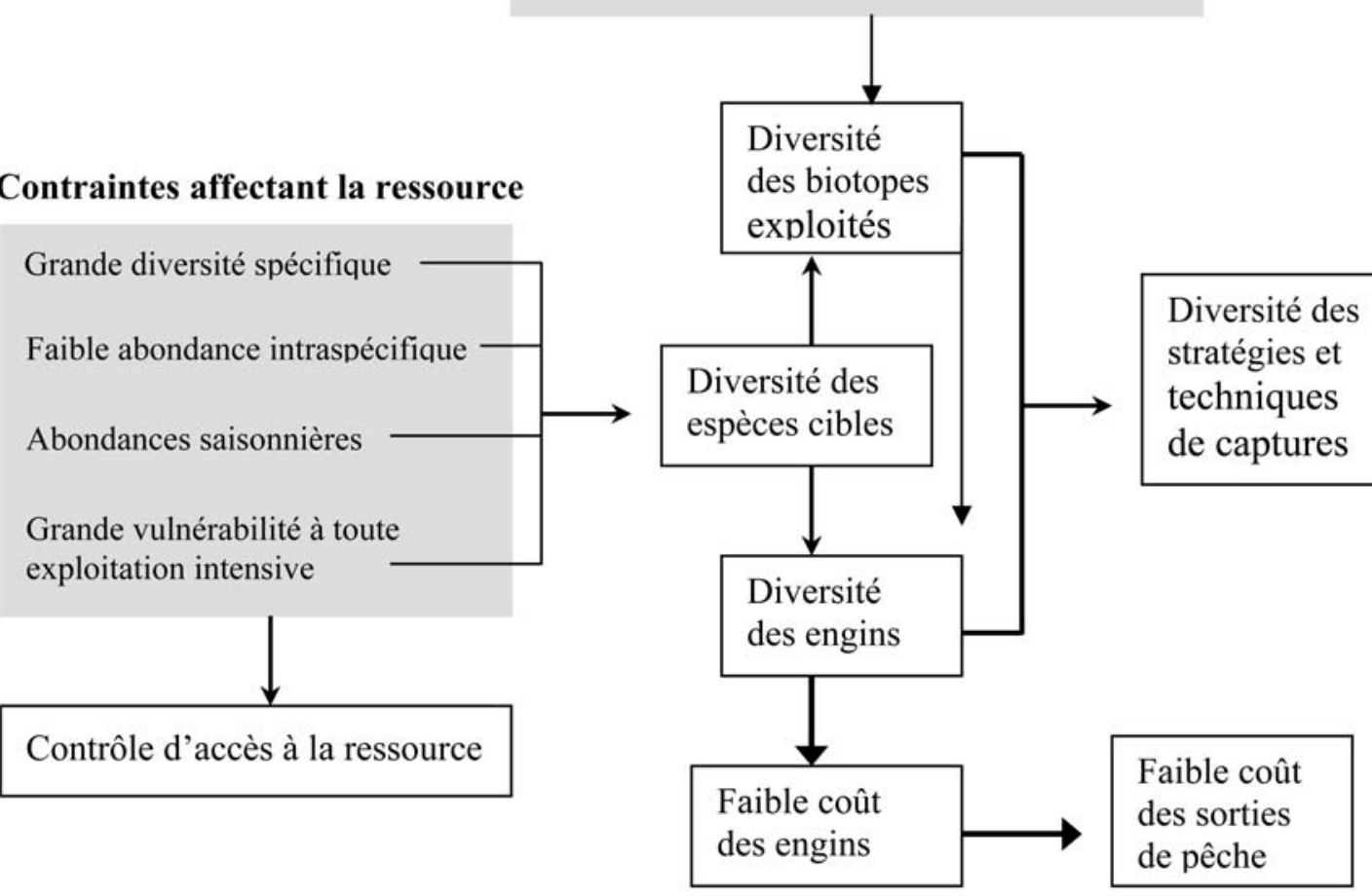

Réponses aux contraintes affectant la ressource

Réponses aux deux contraintes

Réponses aux contraintes affectant la présence des pêcheurs sur les lieux de capture

FIGURE 7. - Adaptation de la pêche villageoise aux contraintes environnementales

afin d'exploiter au mieux un biotope donné. Ainsi la plongée sous-marine peut-elle s'avérer très efficace pour exploiter les premiers mètres du tombant récifal lorsque l'eau est claire et, au contraire, être totalement infructueuse lorsque l'eau est turbide, auquel cas la pêche à la ligne constitue une alternative intéressante. Pour un même type d'engin, il peut donc exister plusieurs techniques de capture ${ }^{12}$ qui varient selon le fait que le pêcheur utilise une embarcation ou n'en utilise pas et selon le biotope exploité ${ }^{13}$. Sur cette base, 97 techniques de captures mettant en œuvre 39 types d'engins ou associations d'engins ont été identifiées lors des 943 sorties de pêche.
Régulation " traditionnelle » de l'activité halieutique

\section{- Situation générale}

Compte tenu de la faible étendue des territoires de pêche et de la sensibilité de la ressource à tout effort intensif, la pêche villageoise est fréquemment confrontée à des menaces de surexploitation en Océanie. Pour perdurer, l'activité halieutique doit être régulée. Dans le cadre de l'économie vivrière traditionnelle (non monétaire), la solution consiste à contrôler l'accès à la ressource par la mise en place d'interdictions temporaires de pêche frappant une partie ou la

12. La technique de capture se définit comme la conjonction d'un mode d'utilisation des engins de pêche (seul ou en association), d'un vecteur de déplacement (à pied, en plongée, embarqué) et d'un biotope exploité.

13. Dans le cas présent, les biotopes ont été regroupés en trois classes : a) le tombant récifal et la zone au-delà ; b) le platier récifal et la zone marine distale formée des herbiers de phanérogames et des plages; c) les embouchures de rivière et les mangroves. Les pêcheurs identifiant dans leurs stratégies de captures un nombre beaucoup plus important de biotopes, le nombre exact de techniques de capture est vraisemblablement plus élevé. 
totalité du territoire de pêche (Dahl, 1988 ; Ruddle, 1988, 1989).

S'appliquant à l'ensemble des membres de la communauté villageoise détentrice du territoire de pêche et aux populations non riveraines ou parfois seulement à ces dernières, ces interdictions temporaires de pêche concernent soit l'ensemble des espèces exploitables, soit les espèces les plus menacées. Elles sont prononcées par le chef du village à l'occasion d'une cérémonie coutumière et donnent souvent lieu à la mise en place d'un signe de reconnaissance, fréquemment un bâton fiché dans le platier, rappelant au passant l'existence du "tabou". La période durant laquelle elles sont en vigueur varie généralement de six mois à trois ans. Tout membre de la communauté enfreignant l'interdit est passible d'une lourde amende coutumière. Lorsque l'interdiction temporaire de capture est levée, l'accès aux zones de pêche redevient de nouveau libre. Pour répondre à l'introduction, durant ces trente dernières années, des fusils sous-marins et des filets maillants, beaucoup plus performants que le matériel de capture «traditionnel», les interdictions temporaires de pêche se doublent parfois de l'interdiction d'usage de ces engins sur les ressources halieutiques. Dans les cas de forte pression, comme dans les atolls de Tonga, de Tuvalu et du sud de Kiribati, la pêche vivrière commerciale de poissons est prohibée et seule la pêche vivrière d'autosubsistance est autorisée (Bataille-Benguigui, 1994 ; Zann, 1989).

L'efficacité de ces interdictions temporaires repose sur la résilience de l'écosystème corallien, qui s'exprime selon deux processus complémentaires concourant à la régénération de stocks halieutiques :

a) la capacité d'une population halieutique, dont la structure par âge a été écrêtée par la surpêche, à se reconstituer du fait du rapide taux de croissance des juvéniles restants. Ainsi, dès que la pression halieutique s'arrête, la proportion d'individus sexuellement matures commence à s'accroître pour atteindre au bout de quelques dizaines de mois un nombre suffisant pour assurer la pérennité du stock, voire une diffusion des jeunes adultes au-delà du territoire de pêche mis en protection ;

b) la «perméabilité » du territoire de pêche aux flux d'œufs ou de larves de poissons, de mollusques et de crustacés venant de l'extérieur qui, " faisant souche », vont reconstituer les stocks locaux.

Ce potentiel de régénération des stocks halieutiques que possède le milieu récifal est un atout considérable qui compense sa sensibilité élevée à toute exploitation intensive; mais toutes les espèces ne présentent pas le même potentiel de recolonisation du milieu. Celles dont les larves vivent en pleine eau sont nettement avantagées par rapport à celles qui passent la majeure partie de leur stade larvaire fixées au fond.

- Quelle régulation pour quelle stabilité ? de la gestion halieutique à la viabilité du territoire

Si la littérature scientifique offre de nombreux exemples de régulation de la pêche vivrière (Johannes, 1978, 1994 ; Carrier, 1981 ; Ruddle et Akimichi, 1984 ; Ruddle et Johannes, 1989 ; South et al., 1994), les synthèses permettant de dégager des caractères communs à l'ensemble des cas d'étude sont rares (Ruddle, 1988 et 1989 ; Polunin, 1989) et il est difficile d'appréhender les logiques d'acteurs sous-tendant ces mécanismes de régulation.

Pour les biologistes s'intéressant au sujet, le maintien ou la restauration de l'abondance récifale constituent l'objectif commun à toute régulation de l'activité halieutique traditionnelle. C'est ce que Johannes $(1978,1981)$ appelle «l'éthique de conservation», qui serait commune à l'ensemble des populations littorales d'Océanie et régirait la gestion traditionnelle des ressources halieutiques. Lorsque dans le cadre de sa thèse, celui-ci travaillait sur la pêche à Palau, cette éthique lui est vite apparue comme une évidence. Sinon comment expliquer que les ressources récifales aient conservé leur qualité après des siècles d'exploitation marqués par des prélèvements intensifs consécutifs aux nombreuses guerres qui affectaient ces îles de Micronésie et aux aléas climatiques qui empêchaient la pêche en haute mer durant de nombreuses semaines? Ces guerres intertribales ont en effet contraint les populations à se retrancher à l'intérieur des terres, mais la pêche est restée leur principale source de protéines. Pour la pratiquer sans courir le risque d'être capturé par les ennemis, l'unique solution consistait à pêcher en groupe, occasionnant ainsi des concentrations importantes de l'effort de pêche. Compte tenu de l'aura dont bénéficiait Johannes dans le Pacifique et de l'antériorité de ses réflexions, le concept de conservation ethic n'a guère été critiqué.

Seul Polunin (1989) fait état de son scepticisme. Dans l'hypothèse selon laquelle le foncier marin et l'éthique de conservation sont étroitement associés, en toute logique, l'absence de l'un devrait traduire l'absence de l'autre. Comment expliquer alors, comme on l'observe en Papouasie Nouvelle-Guinée, que des populations voisines, vivant dans des écosystèmes analogues, présentent l'une une éthique de la conservation 
quand l'autre en est dépourvue? En revanche, souligne cet auteur en s'appuyant sur des exemples indonésiens, le foncier marin semble beaucoup plus souvent avoir été mis en place pour résoudre des conflits d'usages sur les ressources récifales. Cet argument est également avancé par Sims (1989) en ce qui concerne l'atoll de Mangaia aux îles Cook, où une légende recueillie en 1894 fait état de violentes disputes entre deux tribus exploitant un récif adjacent à leur territoire terrestre respectif. Enfin, conclut Polunin, l'examen de la littérature tendrait à donner l'impression que le foncier marin n'est tout simplement que l'extension marine du foncier terrestre, ce que confirme Hviding (1989) pour le lagon de Marovo. Ce point de vue me semble essentiel car il replace l'éthique de la conservation dans un contexte plus global que celui des rapports entre la société et le milieu marin. Ainsi, cette éthique ne reflèterait pas une pratique visà-vis de la gestion des ressources récifales mais serait le produit d'une causalité supérieure : la territorialité, qui irrigue l'ensemble des sociétés océaniennes (David, 1999).

Dans ce contexte, la gestion des ressources récifales est au service de la viabilité du territoire du groupe (tribu, clan famille). Les interdictions temporaires de pêche et le foncier marin qui leur est associé sont le moyen d'en gérer la partie maritime. L'objectif est double : à la fois social et économique. Pour Akimichi (1989), il s'agit principalement d'assurer l'équité sociale et économique dans l'accès à la ressource, et de prévenir les conflits d'usages et des disputes intra et intervillageoises se rapportant à la pêche. À ce point de vue, centré sur les logiques d'acteurs, trois objectifs peuvent être ajoutés. Tous relèvent de la territorialité économique et ne peuvent être remplis que si l'abondance de la ressource halieutique récifale est conservée ou restaurée.

a) Le premier d'entre eux vise à consolider la pérennité de la pêche vivrière en tant que pourvoyeuse régulière de protéines animales. En Mélanésie, cette situation correspond aux littoraux peu propices à l'agriculture car, comme le souligne Bell (1946-47), cité par Polunin (1989), la pêche y est généralement un second choix comparé à cette dernière et ne se pratique avec régularité que lorsque la terre n'est pas assez productive.

b) Le second objectif porte sur le renforcement de la fonction de réserve alimentaire du récif en prévision de « coups durs ». Ce peut être le passage d'un cyclone (cas fréquent en Mélanésie) comme une période prolongée de mauvais temps qui interdit toute sortie en haute mer. Or, dans de nombreux atolls de Polynésie et de Micronésie, notamment à Kiribati et à Tuvalu, celle-ci constitue la principale zone de capture (Zann, 1989) et les pélagiques sont la principale source de protéines des populations.

c) Le troisième objectif vise à fournir une production halieutique suffisante pour les fêtes coutumières ou religieuses dont la fonction est de renforcer la cohésion sociale intracommunautaire ou intercommunautaire. Telle est la situation rencontrée par Hviding (1989) dans le lagon de Marovo, en NouvelleGéorgie (Salomon).

D'un point de vue strictement social, la gestion territoriale des ressources halieutiques permet également d'affirmer l'appartenance du territoire de pêche au territoire du groupe. Cette logique relève d'une territorialité d'essence identitaire, par laquelle le territoire fonde l'identité du groupe ${ }^{14}$ : un lien charnel unit alors l'homme à son territoire, c'est-à-dire à l'espace qu'il s'est approprié pour assurer la reproduction du groupe social auquel il appartient et la satisfaction de ses besoins vitaux.

En définitive, l'outil de régulation de la pêche vivrière océanienne que forment les interdictions temporaires de pêche et le foncier marin qui leur est associé se fonde donc sur une double territorialité (identitaire et économique), qu'exprime parfaitement Hviding (1989 : 26) quand il considère que le foncier marin du lagon de Marovo représente une ressource économique mais aussi une ressource d'histoire et d'identité sans laquelle le clan ne peut exister en tant que groupe.

\section{La pêche artisanale et sa gestion : la gestion halieutique moderne et son application dans le contexte océanien}

Par opposition à la pêche vivrière, gérée de manière "traditionnelle », la pêche artisanale commerciale fait l'objet d'une gestion dite «moderne» puisqu'elle s'appuie sur des connaissances scientifiques et des modèles qui visent à prévoir l'état futur de la pêcherie en

14. Comme l'a montré Bonnemaison (1981 et 1986), les concepts de territoire et d'ethnie sont très imbriqués en Océanie : « L'ethnie se crée et se conforte par la profondeur de son ancrage au sol, et par le degré de correspondance plus ou moins élaboré qu'elle entretient avec un espace qu'elle structure, quadrille et polarise selon ses propres finalités et représentations symboliques : son territoire. » 
fonction de l'état de la ressource et de la pression de pêche qui s'exerce sur elle. Gérer la pêche revient donc à déterminer un niveau acceptable de mortalité par pêche des stocks de poissons, compte tenu de leur dynamique démographique (Brêthes, 2000).

Au quotidien, en Océanie, la gestion moderne de la pêche commerciale repose sur la collecte régulière et le traitement informatique de statistiques concernant l'effort de pêche, le poids et la taille des captures (Gulland, 1983 ; Polovina et Ralston, 1987 ; Moffit, 1993) afin d'estimer la quantité maximale de poissons qui peut être capturée chaque année, la prise maximale équilibrée (PME), sans compromettre les capacités de renouvellement du stock. La PME est généralement définie pour l'ensemble d'un pays, plus rarement région par région. Lorsque des données collectées le permettent, la précision de l'estimation peut descendre au niveau de l'île (Cillaurren et al., 2001). Disposant de la PME et du rendement moyen des embarcations, les décideurs établissent le bilan des entrées et des sorties dans la flottille de pêche. Lorsque l'effort de pêche est bien inférieur à celui requis pour atteindre la PME, une assistance financière et technique est accordée aux pêcheurs pour accroître le nombre d'embarcations et l'effort de pêche (Cillaurren et David, 1995). Dans le cas contraire, les pêcheurs sont invités à reporter leur effort vers d'autres ressources, notamment les poissons pélagiques agrégés autour des dispositifs de concentration de poissons (DCP). En définitive, bien qu'elles reposent sur la connaissance scientifique de la ressource et l'emploi de modèles mathématiques, ces mesures de gestion restent encore trop globales et trop théoriques et l'on peut légitimement se demander si "l'appareillage » scientifique mis en œuvre n'est pas disproportionné par rapport aux résultats attendus.

En effet, si les modèles classiques de type Schaeffer (1954) utilisés pour définir la PME dans la gestion des pêches commerciales et les modèles bio-économiques qui en sont dérivés (Gordon, 1954 ; Gilly, 1989 ; Reveret, 1991) apparaissent assez bien adaptés aux exploitations monospécifiques, ils s'appliquent beaucoup plus difficilement aux pêcheries multi-spécifiques. La gestion des stocks fait alors appel à des modèles analytiques qui intègrent les paramètres biologiques (recrutement, croissance et mortalité) de chaque espèce pour tenter de donner une estimation du taux limite d'exploitation basé sur une capture optimale de chaque espèce (Laurec et Le
Guen, 1981). Les années 1950 à 1980 ont vu se développer une complexification croissante de cette modélisation à but quantitatif, reposant sur un postulat d'homogénéité de répartition spatiale de la ressource halieutique. Or, la structure géologique des archipels océaniens conduit à un éclatement de cette ressource en de nombreux microstocks aux dynamiques démographiques spécifiques, ce qui rend la collecte des données difficile et coûteuse (Cillaurren et David, 2000).

Le questionnement est encore plus pertinent lorsqu'il s'applique aux pêcheries récifales, caractérisées par une forte dispersion géographique et l'extrême diversité des espèces cibles qui rendent non opérationnels les modèles classiques en vigueur. Ainsi en 2000, lors de l'atelier régional de l'ICRI (International Coral Reef Initiative) dans le Pacifique Sud, Johannes et al. (2002) se sont faits les apôtres de ce qu'ils appellent « une gestion à données minima » ${ }^{15}$ prenant acte « du décalage énorme entre le coût des programmes de collecte des données menées dans la plupart des pays en développement et les moyens plus modestes qu'il suffirait de mettre en œuvre pour prendre des décisions efficaces, tant en matière de surveillance que de gestion $»$. Comme le soulignent ces auteurs dont T. Adams, actuel directeur de la division des ressources marines de la CPS :

« [...] il n'y a guère intérêt à essayer de mettre en place des modèles de gestion à l'occidentale, qui ne peuvent fonctionner que s'ils s'appuient sur un immense volume de données scientifiques et ne s'appliquent qu'à des pêcheries commerciales de type occidental... nous avons désespérément besoin de solutions qui soient insensibles aux défaillances éventuelles du système, des solutions qui nous permettront de nous prémunir contre les conséquences d'évaluation inexactes des stocks, du manque de données fondamentales sur l'environnement ou encore d'une répartition trop optimiste de l'effort de pêche. »

Pour l'instant ce mode de gestion à données minima reste encore au stade des idées. Aucune expérience d'envergure n'a encore été tentée en Océanie. On notera cependant qu'à Vanuatu la diffusion généralisée de planches à mesurer les poissons auprès des pêcheurs participant au programme de recherche de l'IRD sur les principales espèces démersales profondes de 1983 à 1992 a permis de prendre conscience que la mesure de la taille des captures est un moyen simple pour les pêcheurs de gérer leur ressource halieutique. Dès que les prises sont inférieures à la taille minimale

15. Cette traduction me semble préférable à celle adoptée par le service de la Traduction de la CPS : " gestion sans donnée ». L'expression anglaise originelle est en effet data less management qui ne signifie pas qu'on se prive de données mais qu'on utilise d'autres informations que les données classiques de prises et d'effort. 
requise par les scientifiques (généralement celle-ci correspond au stade de maturité sexuelle), l'effort se déplace sur d'autres zones de pêche de manière à laisser au stock le temps de se reconstituer ou du moins aux juvéniles de devenir adultes et de se reproduire au moins une fois.

La réflexion sur la gestion des stocks halieutiques à données minima n'est pas spécifique du Pacifique Sud. Dans l'océan Indien, une approche similaire menée par l'Institut des Ressources Marines de l'Université de Dar es Salaam à Zanzibar vient d'aboutir à l'élaboration d'une méthode d'évaluation et de gestion participatives des stocks halieutiques, axée sur la prise en compte du savoir des pêcheurs et sur leur participation à la collecte de données (Walmsley et al., 2005). La méthode se développe en six étapes : a) la compréhension du contexte halieutique, b) l'accord avec les pêcheurs sur les objectifs à suivre, c) la mise en œuvre de l'évaluation participative des stocks, d) l'interprétation des résultats et la restitution auprès des pêcheurs, e) les premières mesures de gestion, f) l'évaluation du processus (figure 8). Cette méthode semble très prometteuse et offre de nouvelles perspectives quant à l'implication des pêcheurs dans la gestion des stocks halieutiques et la pérennisation d'un système efficace et peu coûteux de collecte de l'information sur les pêcheries côtières. Toutefois, même s'ils ne sont pas impliqués par les pouvoirs publics dans ces processus de gestion à données minima des ressources halieutiques, les pêcheurs peuvent être actifs en matière de gestion.

S'inspirant des modes traditionnels en vigueur pour la pêche vivrière, certains d'entre eux appliquent une logique "d'ouverture et de fermeture » de l'espace qui, pour l'instant, se limite à interdire l'accès des zones de pêche aux embarcations venant des villages voisins. Il ne s'agit donc pas à proprement parler d'une gestion de la ressource mais plutôt de la construction d'un territoire que les pêcheurs s'approprient progressivement par leurs pratiques halieutiques et l'exclusivité d'usage qu'ils se sont arrogés au détriment de leurs voisins et à l'encontre des juridictions en vigueur qui toutes prônent l'accès libre aux ressources de la mer territoriale pour les nationaux. Lorsque ce territoire halieutique est reconnu comme tel par les communautés littorales, une gestion plus fine de la ressource peut être envisagée. Ainsi à Santo, île du nord de Vanuatu, les pêcheurs ont du faire face à une surexploitation de Pristipomoïdes multidens, espèce localisée autour de $150 \mathrm{~m}$ de profondeur, qui s'est traduite par une diminution drastique de la taille des captures. Deux comportements adaptatifs étaient possibles: soit changer de champs de pêche en gardant la même espèce cible, ce qui est la démarche logique dans un contexte de libre accès à la ressource, l'effort de pêche se déplace ainsi dans l'espace à chaque fois que des signes de surexploitation se manifestent, soit rester sur les mêmes zones de pêche et diversifier les profondeurs de prise afin de capturer d'autres espèces, même si celles-ci sont d'un moindre intérêt commercial. C'est cette dernière logique qui a été suivie (Cillaurren et Simier, 1999). Elle peut être interprétée comme l'émergence d'un concept de patrimoine halieutique, fruit de la relation qui lie le pêcheur, la ressource et le territoire de pêche (Cillaurren et David, 2003).

Prenant acte de la volonté des pêcheurs de s'impliquer dans la gestion de leurs ressources halieutiques et de l'inefficacité globale du modèle de gestion de la pêche artisanale océanienne mis en place par les administrations nationales des pêches avec l'appui des organisations internationales (Communauté Pacifique, Agence des pêches du Forum du Pacifique Sud, FAO) ${ }^{16}$, certains scientifiques (Dahl, 1988 ; Sims, 1989 ; Johannes, 1994, 1998), relayés désormais par des institutions internationales comme la CPS, proposent de laisser la gestion de la ressource aux villageois en revitalisant les règles ancestrales pour les transformer en un mode de co-gestion applicable à l'échelon local comme au niveau national, faisant ainsi des aires marines protégées un nouvel outil de la gestion des ressources halieutiques. C'est cette nouvelle évolution que nous allons maintenant examiner.

\section{Les aires marines protégées villageoises, nouvel outil de gestion des ressources halieutiques océa- niennes?}

S'appuyer sur les règles ancestrales de gestion des territoires de pêche pour élaborer un mode de co-gestion des ressources halieutiques à l'échelon du village comme à celui du pays est une idée novatrice :

a) ce n'est plus une ressource qui est gérée mais l'espace où celle-ci vit, considéré comme un patrimoine dont la bonne gestion à l'échelle locale garantit la pérennité du patrimoine national, composé de l'ensemble des patrimoines locaux ;

16. Même lorsque des données fiables peuvent être recueillies comme cela a été le cas à Vanuatu, les mesures de gestion qui en résultent sont peu efficaces, car inapplicables à l'échelle locale, faute de représentants de la force publique sur place. 


\begin{tabular}{|c|c|}
\hline $\begin{array}{l}\text { 5. Mesures de gestion } \\
+ \text { Construction d'un consensus } \\
\text { sur les mesures à prendre } \\
+ \text { Élaboration du plan de gestion } \\
\text { et des mesures à appliquer } \\
\text { + Définition des critères de } \\
\text { suivi-évaluation }\end{array}$ & $\begin{array}{l}\text { 4. Interprétation et restitution } \\
\text { des résultats } \\
\text { + Interprétation des résultats de } \\
\text { l'évaluation } \\
\text { + Restitution auprès des } \\
\text { pêcheurs et des autorités }\end{array}$ \\
\hline $\begin{array}{l}\text { 6. Évaluation du processus } \\
+ \text { Évaluation de l'impact des } \\
\text { mesures de gestion } \\
+ \text { Évaluation de la collecte de } \\
\text { l'information et de l'évaluation } \\
\text { participative des stocks } \\
\text { + Évaluation du processus } \\
\text { participatif }\end{array}$ & $\begin{array}{l}\text { 3. Mise en œuvre de } \\
\text { l'évaluation des stocks } \\
\text { + Définition des besoins } \\
\text { en information } \\
\text { + Collecte des données } \\
\text { + Analyse des données } \\
\text { collectées }\end{array}$ \\
\hline $\begin{array}{l}\text { 1. Compréhension du contexte } \\
\text { + Évaluation de la pêcherie et de } \\
\text { son environnement de gestion } \\
\text { + Identification des pêcheurs à } \\
\text { impliquer } \\
\text { + Identification des moyens de } \\
\text { communication appropriés }\end{array}$ & $\begin{array}{l}\text { 2. Accord avec les pêcheurs sur } \\
\text { les objectifs à suivre } \\
\text { + Mobilisation des pêcheurs } \\
\text { + Explication quant à la méthode } \\
\text { à suivre } \\
\text { + Définition conjointe des } \\
\text { objectifs de gestion }\end{array}$ \\
\hline
\end{tabular}

FIGURE 8. - Les étapes de la méthode d'évaluation participative des stocks halieutiques (d'après Walmsley et al., 2005)

b) les règles de gestion ne s'appuient plus sur des modèles mathématiques mais sur une décision collective qui, prônant l'ouverture ou la fermeture de l'espace-ressource à la pêche, est prise au niveau du village. Lorsque cette mise en réserve du territoire de pêche villageois est prise de concert ou en accord avec les autorités nationales, on peut parler de cogestion ou « collaborative management » (Sen et Nielsen, 1996 ; Barrow et al., 2000 ; Nurse et Kabamba, 2000,). Issu des études sur la gestion des forêts en Asie (Gilmour, 1990; Fisher, 1995), ce concept suppose toujours l'implication très forte des populations locales dans la gestion des ressources qu'elles exploitent, que l'on qualifie de " gestion participative » ou « community-based management ».

Cette idée de co-gestion par l'État et les populations locales d'aires marines protégées (AMP) rejoint les préoccupations défendues par un nombre croissant de biologistes des pêches qui à l'instar de D. Pauly (1997 : p. 40) considèrent les AMP comme «... les seules approches éprouvées pour assurer la survie des espèces à longue vie présentant de faibles zones d'extension». Elles seules permettent de préserver la complexité des écosystèmes marins et de repeupler progressivement les zones altérées par la pression halieutique.

Mettre en place une co-gestion des ressources halieutiques à l'échelon local en s'appuyant sur les règles ancestrales de gestion et en les redynamisant repose sur le postulat selon lequel ces dernières sont supérieures aux modes de gestion modernes puisqu'elles ont montré par le passé leur aptitude à sauvegarder le milieu naturel et les ressources exploitables qu'il abrite. Mais cette supériorité risque bien de n'être qu'historique et inadaptée aux conditions dans lesquelles vivent 
les populations actuelles. Il est, en particulier, nécessaire de s'interroger sur l'efficacité de ces règles de gestion dans un contexte insulaire de plus en plus marqué par la globalisation. Les communautés locales ont beaucoup évolué durant les deux derniers siècles (David, 2003). Les systèmes fermés qui vivaient en autarcie se sont ouverts, l'argent circule jusque dans les lieux les plus reculés et l'univers mental des populations s'est transformé. Face à ces changements, il est illusoire de croire que les modes de gestion fonctionneront correctement dans le futur sur la seule base qu'ils ont bien fonctionné dans le passé. Les difficultés de gérer de manière durable les pêches aux coquillages nacriers (trocas et burgaux) et aux holothuries dans les archipels du Pacifique Sud en offrent un bon exemple.

Les trocas (trochus niloticus), les burgaux (Turbo marmoratus) et les holothuries (bêches de mer) font l'objet d'une pêche commerciale en Océanie depuis la première partie du $\mathrm{XIX}^{\mathrm{e}}$ siècle. Au même titre que les baleines, ils ont participé à l'insertion de cette région dans l'économie mondiale, à telle enseigne que les sabirs parlés au Vanuatu, aux Salomon et en Papouasie Nouvelle-Guinée sous le nom de « bichlamar » ou de « pidgin » ont été forgés pour faciliter leur commerce. Cette exploitation s'est pratiquée dans le cadre d'une économie de prédation, les « beach combers » écumant une zone avant de passer à une autre. Ce mode d'exploitation semble toujours en vigueur au regard des statistiques de production des holothuries en Océanie : un pays demeure leader des exportations durant deux à trois ans avant de laisser la place à un autre pays, ses stocks ayant été sérieusement entamés et non renouvelés, faute de gestion. Ainsi à Fiji, de 1984 à 1988, les exportations de bêches de mer sont passées de $50 \mathrm{t}$ à plus de $700 \mathrm{t}$ pour retomber à moins de $300 \mathrm{t}$ en 1990. C'est alors que la forte demande sur le marché international a conduit à la «montée en puissance» des îles Salomon dont les exportations d'holothuries, après avoir stagné entre 100 et 150 t de 1986 à 1990, se sont élevées en 1991 à plus de $600 \mathrm{t}$ pour culminer à 715 t en 1992 puis ensuite s'effondrer (Bettencourt, 1995).

Si les holothuries étaient gérées à «l'équilibre » dans un cadre villageois, de telles évolutions seraient impossibles. C'est le pari tenté aux îles Moluques dans l'est de l'Indonésie avec l'exploitation des trocas. D'anciennes interdictions coutumières ont été remises en vigueur au début des années 1980. La pêche n'est désormais ouverte que 2 à 3 jours par an et donne lieu à une cérémonie, le Sasi, dont l'objectif est de rappeler la légitimité des chefs coutumiers locaux à gérer la ressource. Cet exemple d'AMP villageoise dédiée à une ressource commerciale hautement prisée sur le marché international est souvent présenté comme la preuve de la « sagesse » des modes de gestion traditionnelle guidée par une éthique de la conservation. Or la réalité décrite par I. Antunès (2000) dans le cadre de sa thèse sur le village de Watlar dans l'île Key Besar (partie orientale des Moluques) est bien différente. En préservant le stock de trocas, il s'agit avant tout de maximiser les revenus monétaires tirés de leur exploitation, les autres ressources du village n'offrant guère de possibilités de commercialisation. Cette maximisation des revenus monétaires est le moyen d'assurer la viabilité du socio-système et du territoire qui le porte. Tant que la préservation de la ressource halieutique concourt à la viabilité territoriale, la pêche est gérée correctement, laissant à penser qu'une éthique de conservation la préside. Mais dans le cas contraire que se passerait-il ?

La réponse se trouve dans les archipels d'Océanie où, pour satisfaire les marchés internationaux du trocas et des holothuries, tous deux marqués par une forte demande (Conand, 1986 ; Bour, 1990), des acheteurs circulent jusque dans les îles les plus reculées, proposant aux communautés locales des sommes considérables au regard de leur pouvoir d'achat. Il est fréquent que celles-ci acceptent, faisant courir le risque d'une surexploitation au stock halieutique. $\mathrm{Ce}$ comportement, diront certains, est le signe de l'érosion de "l'éthique de conservation ». Pour ma part, je considère qu'il s'agit d'une démarche logique qui vise à améliorer la viabilité du territoire villageois ${ }^{17}$. Celle-ci recouvre une double dimension: d'une part, la satisfaction des besoins des familles, d'autre part la fourniture de services communautaires par la collectivité villageoise. Pour les chefs coutumiers, sollicités par les acheteurs de trocas, comment résister à cette manne dans le contexte général du désengagement financier des États, qui n'assurent plus que trop rarement les réparations des infrastructures de santé, d'éducation et de transport ? Que pèse alors, face à l'amélioration du bien-être de la communauté, la menace d'une surexploitation des stocks halieutiques? À la différence de la pêche vivrière, la pêche artisanale commerciale

17. Doumenge (1983) définit la viabilité comme la combinaison d'un état statique, « la réunion des conditions nécessaires et suffisantes pour exister et durer », et d'un état dynamique assimilable aux conditions « à réunir pour pouvoir se développer tant sur le plan de l'utilisation la plus complète des ressources naturelles que pour la progression du niveau de vie social et économique de la population ». 
ne génère pas de territorialité identitaire. Le territoire de pêche est une ressource économique. Dans le cadre de cette territorialité économique, il peut être rationnel de surexploiter les stocks halieutiques les plus recherchés sur le marché international si les revenus qui en sont tirés permettent d'accroître la viabilité globale du territoire villageois en finançant, par exemple, la rénovation d'un dispensaire ou l'agrandissement de l'école.

Outre le support d'une territorialité économique, les AMP villageoises sont également souvent un espace de régulation, mis en place pour résoudre les conflits opposant deux groupes voisins pour l'exploitation d'une ressource qui jusqu'alors était libre d'accès, car considérée comme sans valeur dans le système économique traditionnel. Cette fonction n'est donc pas au service de l'écosystème mais à celui des communautés villageoises.

Qu'elles soient dédiées à la pêche vivrière ou à la pêche commerciale, les AMP traditionnelles sont gérées par le système politique traditionnel (chef coutumier, conseil des grands hommes). La perte d'autorité de ce dernier pose la question de l'efficacité de cette gestion face aux deux problèmes majeurs que sont l'accroissement démographique, qui génère une pression accrue sur les ressources vivrières, et la forte demande sur le marché international d'holothuries et de coquillages nacriers rapportée à l'appauvrissement grandissant de nombre d'économies villageoises insulaires (figure 9). Il est donc permis d'être réservé quant à la capacité des communautés locales à résister aux sollicitations monétaires dont elles sont parfois l'objet, pour une exploitation non pérenne de leurs ressources récifales, dans un contexte marqué par l'ouverture des villages sur l'économie monde et par le désengagement de l'action publique en zone rurale. Certaines communautés locales ont cette capacité, d'autres l'ont perdue. Si un service des pêches s'engage dans la mise en place d'aires marines protégées sous un régime de co-gestion avec une majorité de villages qui sont dans ce dernier cas, la probabilité est forte que le programme se solde par un échec. La difficulté à laquelle sont confrontés les pouvoirs publics désireux de promouvoir les AMP à l'échelon local tient à l'hétérogénéité spatiale des situations. Deux villages voisins peuvent présenter deux aptitudes tout à fait différentes à gérer leurs ressources. Il convient donc de tester toutes les communautés villageoises avant de s'engager dans un programme de ce type afin de sélectionner les plus aptes à gérer leurs ressources halieutiques. Ce test se fait sous la forme d'enquêtes qui permettent d'élaborer des indicateurs d'aptitude à gérer les ressources récifales à l'échelle locale (figure 10).

Quatre types d'indicateurs peuvent être établis ; les deux premiers mesurent les capacités de la communauté à affronter les sollicitations commerciales extérieures. Ils portent respectivement sur :

a) la cohésion sociale du village ;

b) l'autorité des chefs coutumiers ;

c) l'implication du gouvernement national à l'échelle locale, notamment en matière de santé, d'éducation et de transport ; plus cette implication sera faible, plus sera grand le risque que les communautés locales cèdent aux sollicitations des acheteurs de produits halieutiques ;

d) la valeur sur le marché mondial des produits de la pêche susceptibles d'être commercialisés ; plus cette valeur est élevée, plus les communautés locales seront sollicitées.

Ces indicateurs peuvent être représentés de manière graphique sur quatre axes gradués selon deux niveaux de valeur : faible, élevé. Dans la figure 10, les deux cas opposés sont représentés : la bonne et la mauvaise aptitude à gérer les ressources récifales.

\section{Conclusion}

De toutes les activités d'exploitation des ressources vivantes, la pêche est probablement celle qui est la plus marquée par la dialectique instabilité/stabilité. Elle se déroule en effet dans un environnement particulièrement instable. Les conditions climatiques et les difficultés du travail à la mer, qui conditionnent la présence du pêcheur sur les lieux de pêche, sont en effet soumises à une grande variabilité temporelle dont les causes échappent largement à l'entendement des pêcheurs et dont ils sont donc incapables de prévoir les tendances au-delà de la journée. Cette vulnérabilité aux rythmes qu'impose le milieu naturel est également patente en ce qui concerne la biologie et l'éthologie des espèces exploitées qui conditionnent la présence du poisson sur les lieux de pêche. À l'instabilité de l'environnement physique et biologique de la pêche s'ajoute l'opacité du milieu aquatique qui confère à cet environnement un caractère hostile qui accroît d'autant la vulnérabilité du pêcheur vis-à-vis des aléas naturels. Pour mettre des chances de son côté, le pêcheur peut s'adonner à la prière ou à tout autre rituel religieux permettant de réduire cette instabilité s'il considère que cette dernière 


\section{Satisfaire les pré-requis}

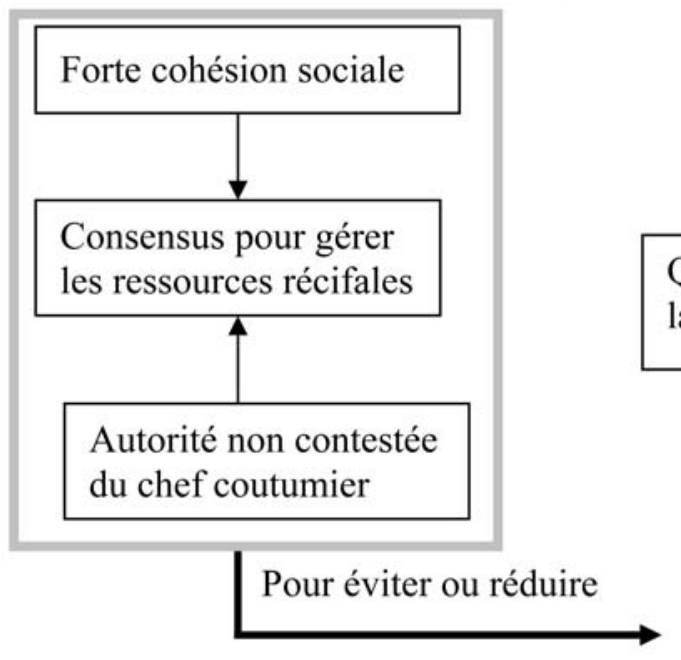

\section{Minimiser les contraintes suivantes}

Valeur commerciale élevée sur le marché mondial (holothurie, coquillages nacriers, poissons d'aquarium)

Quantité d'argent importante proposée aux villageois comparée aux autres sources de revenus

Insatisfaction des villageois et tentation de surexploiter leurs ressources récifales afin de disposer de revenus suffisants pour améliorer leur bien être en matière d'éducation, de santé et de transport.

Faible implication du gouvernement national à l'échelle locale

FIGURE 9. - Conditions à réunir pour gérer durablement les ressources récifales à l'échelle villageoise

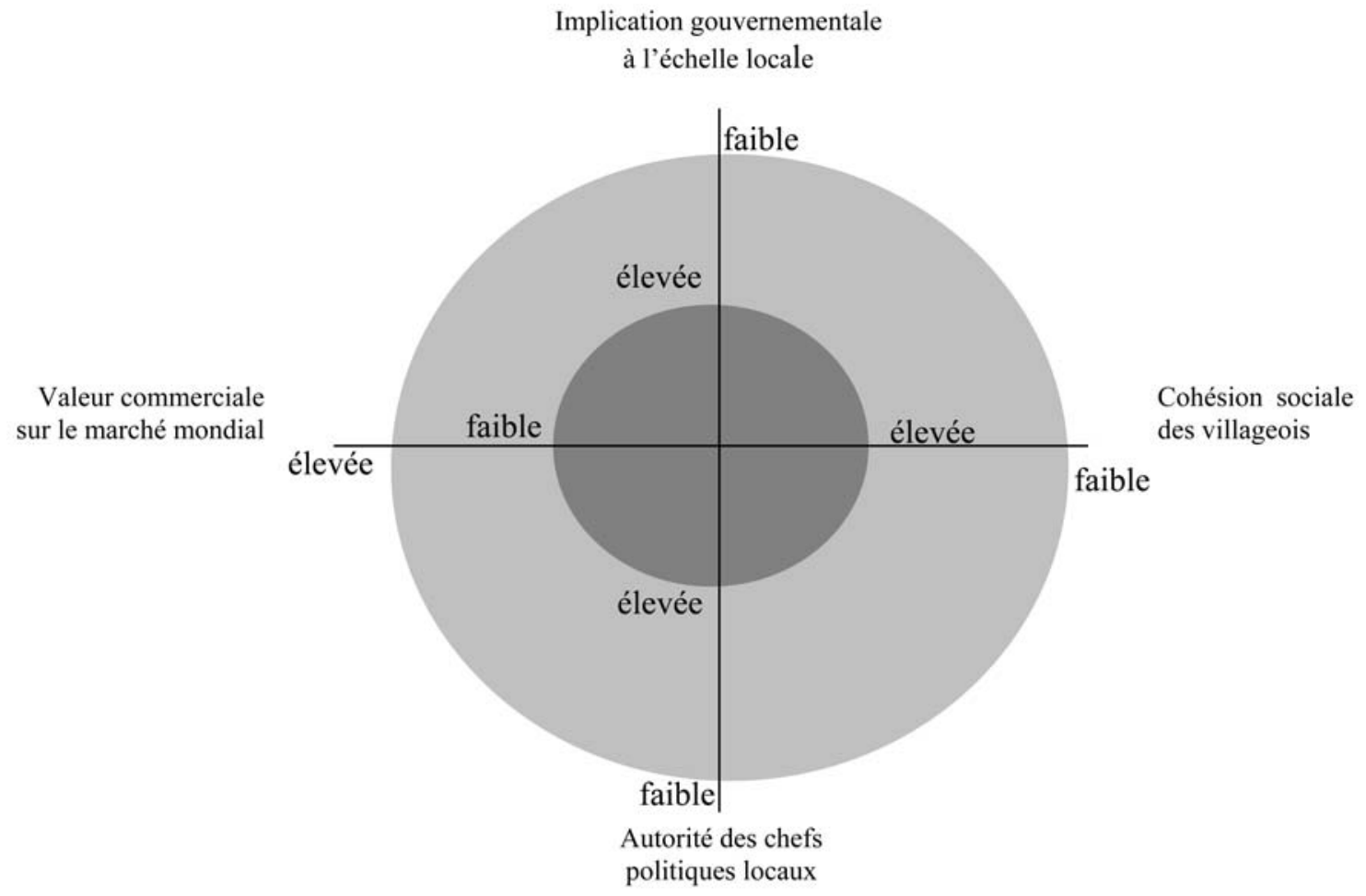

Bonne aptitude, capacités intrinsèques maximales, contraintes minimales

Mauvaise aptitude, capacités intrinsèques minimales, contraintes maximales

FIGURE 10. - Indicateurs d'aptitude à gérer les ressources à l'échelle locale 
est d'origine cosmique. Il peut également s'attacher à diminuer sa vulnérabilité aux contraintes naturelles en adoptant des stratégies de pêche, qui s'expriment à l'échelle de la sortie de courte durée ou de la saison de pêche en termes de biotopes prospectés et d'engins utilisés. Lorsque l'espace où les poissons, les coquillages ou les crustacés peuvent être capturés n'est pas extensible, le pêcheur doit se muer en gestionnaire de la ressource pour que l'activité halieutique puisse perdurer. Si pêcher est souvent un acte individuel, qui peut se ramener à une relation écologique de type " prédateur-proie », gérer est toujours un acte collectif. En ce sens, la gestion s'inscrit principalement dans le champ des relations sociales même si sa finalité vise à réguler la pêche individuelle. En Océanie, la gestion halieutique traditionnelle s'exprime sous une forme territoriale. Au lieu d'agir sur la mortalité par pêche, paramètre central de la dynamique des populations exploitées sur laquelle s'appuie la gestion moderne des pêches, elle se contente de définir des règles d'accès et d'interdits au territoire de pêche, ce qui de fait instaure des jachères halieutiques durant lesquelles les biocénoses perturbées sont censées recouvrer une nouvelle stabilité. À la différence de la gestion "moderne» des pêches qui requiert des données régulières sur les prises (taille et poids des espèces pêchées) et sur les activités à la mer (effort de pêche) pour nourrir des modèles mathématiques permettant de prédire l'évolution quantitative des populations exploitées, ce type de gestion ne nécessite aucune information concernant les ressources marines. Aussi de nombreux services des pêches de l'Océanie s'interrogent sur l'opportunité de s'inspirer de ces modes de gestion traditionnels pour bâtir un système de gestion des ressources halieutiques exploitées par la pêche artisanale qui soit beaucoup moins coûteux et nettement plus simple à mettre en place et à entretenir au quotidien que les systèmes de collecte de données requis pour une gestion "moderne » des pêches. Dans ce contexte, dans le Pacifique comme ailleurs, les aires marines protégées sont en passe de devenir des outils de gestion des ressources halieutiques en plaçant le territoire au centre de la régulation des activités de pêche. On revient ainsi au modèle ancestral océanien, redynamisé par de nouvelles pratiques comme la cogestion et la gestion participative.

Toutefois, leur nouveauté et l'intérêt qu'elles suscitent pour réduire l'instabilité inhérente aux rapports entre les hommes et leur environnement naturel ne doivent pas occulter le fait que, en tant que mode de gestion des pêcheries, les aires marines protégées reposent sur des processus sociaux complexes. Ce n'est plus d'une relation de type prédateur-proie entre l'homme et une ressource halieutique dont il est question mais de relations entre différents groupes d'hommes et leur territoire ou un territoire en devenir qu'ils doivent s'approprier. Or, toute gestion environnementale fondée sur la création de territoires induit de nouveaux rapports sociaux et de nouveaux conflits d'usage. L'introduction de la territorialité dans la gestion halieutique confère donc à cette dernière un surcroît de complexité, et d'instabilité si à la territorialité se greffent des enjeux de type identitaires. Dans ce contexte, la croissance démographique, la globalisation de l'économie et les changements climatiques qui s'annoncent laissent de grandes interrogations sur la capacité future des pêcheurs côtiers océaniens à maîtriser l'instabilité grandissante qui menace leur activité, voire leur genre de vie.

\section{BIBLIOGRAPHIE}

Aкімісні T., 1989. Territorial regulation in the smallscale fisheries of Itoman, Okinawa, in RUDDLE D. and R.E. JoHANnEs (eds.), Traditional marine resource management in the Pacific basin : an anthology, Jakarta, UNESCO/ROSTEA, pp. 123-158.

ANELl B., 1955. Contribution to the history of fishing in the southernseas, Upsala, Studia Ethnographia Upsaliensa IX, 249 p.

AntunÈs I., 2000. Le développement local de la pêche en Indonésie, entre unité politique et diversité culturelle, une approche à partir de deux situations contrastées: Bendar à Java et Watlar aux Molluques, Paris, Université de Paris IV-Sorbonne, thèse de doctorat de géographie culturelle, $487 \mathrm{p}$.

Barrow E., Gichoni H. and M. Hinfield, 2000. Summary and key lessons from a comparative review of analysis of community conservation in East Africa, Nairobi, IUCN, 46 p.

Bataille-Benguigui M.-C. (ed.), 1981. La pêche traditionnelle en Océanie, Journal de la Société des Océanistes 72-73, pp. 143-313.

_, 1994. Le côté de la mer, quotidien et imaginaire aux îles Tonga Polynésie occidentale, Bordeaux, CRET, coll. Iles et archipels, $321 \mathrm{p}$.

BAtтı A., 2005. Spatialisation des pluies extrêmes et cartographie de l'aléa " érosion des sols » dans les bassins versants en amont du lagon de St Gilles (île de la Réunion), Montpellier, ENGREF/EnsAM/ IGEN/INA Paris-Grignon, mémoire de Master sILAT, $54 \mathrm{p}$.

BELL F.L.S., 1946-1947. The place of food in the social life of the Tonga, II. The art of fishing, Oceania 17, pp. $310-326$. 
Bettencourt S., 1995. Pacific island economies : sustainable management of fisheries, Washington, World Bank.

Blanchet G. et G. Borel, 1988. The "Poti Marara" or the success of a small fishing-boat fitted to needs, Proceedings of the world symposium on fishing gear and fishing vessel design, St John's Newfoundland (Canada), pp. 244-248.

Blanchet G., Borel G. et J. Poanfate, 1987. Petite construction navale et pêche artisanale en Polynésie française, Tahiti, ORSTOM, Notes et documents océanographiques $34,99 \mathrm{p}$.

Blanchet G., Caillaud 1. et J. Poaafate, 1985. Un aspect de la pêche artisanale en Polynésie Française : les pièges à poissons de Tikehau, Tahiti, Orstom, Notes et documents océanographiques 25, $116 \mathrm{p}$.

Bonnemaison J., 1981. Voyage autour du territoire, L'Espace géographique 4, pp. 249-262.

-, 1986. Les fondements d'une identité : territoire, histoire et société dans l'archipel de Vanuatu (Mélanésie). Livre 1 L'arbre et la pirogue, Paris, orstom, coll. Travaux et documents 201, 540 p.

Bour W., 1990. Les ressources halieutiques des pays insulaires du Pacifique. Troisième partie : les trocas, Rome, FAO, doc. tech. sur les pêches 272.3, 89 p.

BRÊTHES J.C., 2000. Spatialiser la gestion : mortalité globale et mortalité locale, in Gascuel D., Chavance P., Bez et N.A. Biseau (éds), Les espaces de l'halieutique, Paris, IRD, coll. Colloques et séminaires, pp. 567-579.

CARrier J.G., 1981. Ownership of productive resources on Ponam island, Manus Province (PNG), Journal de la Société des Océanistes 72-73, pp. 205-217.

Cillaurren E., 1999. La pêche autour des dispositifs de concentration de poissons à Vanuatu, in G. Blanchet (ed.), Les petites activités de pêche dans le Pacifique Sud, Paris, IRD, pp. 145-159.

Cillaurren E. et G. David, 1995. Dynamique de l'exploitation d'une ressource halieutique vierge en économie insulaire peu monétarisée, Questions sur la dynamique de l'exploitation halieutique, table ronde ORSTOM/IFREMER, Montpellier, 6-8 septembre 1993, Paris, ORstom, coll. Colloques et séminaires, pp. 433 - 487.

—, 2000. Hétérogénéité spatiale du système pêche et structuration d'un système d'information pour gérer la ressource : l'exemple du Vanuatu, archipel océanien, Les espaces de l'halieutique, IRD, coll. Colloques et séminaires, pp. 527-548.

_, 2003. Le patrimoine, nouveau concept de gestion halieutique au service du développement durable? l'exemple des ressources côtières en Océanie, in Cosaert P. et F. Bart (éds.), Patrimoines et développement dans les pays tropicaux, Bordeaux, Dymset, coll. Espaces tropicaux 18, pp. 279-288.

Cillaurren E., David G. et R. Grandperrin, 2001. Atlas des pêcheries côtières de Vanuatu, un bilan décennal pour le développement, Paris, IRD, 256 p.
Cillaurren E. et M. Simier, 1999. Spatial constraints in the management of deep demersal fisheries in Vanuatu, Spatial Processes in fisheries, Alaska Sea Grant Symposium, 27-30 october 1999, Anchorage, University of Alaska, Sea Grant College Program, $10 \mathrm{p}$.

ConAND C., 1986. Les ressources halieutiques des pays insulaires du Pacifique. Deuxième partie: les holothuries, Rome, FAO, Doc. tech. sur les pêches 272.2, $108 \mathrm{p}$.

Conand F. 1987. Biologie et écologie des poissons pélagiques du lagon de Nouvelle-Calédonie utilisables comme appât thonier, Brest, Skol Veur Breizh Izel, thèse de doctorat d'État, $235 \mathrm{p}$.

DAHL A.L., I988. Traditional marine tenure : a basis for artisanal fisheries management, Marine Policy 12, pp. 40-48.

Dalzell P. and T. AdAms, 1994. The present status of coastal fisheries production in the South Pacific Islands, 25 regional Technical Meeting on Fisheries, WP 8, Noumea, South Pacific Commission, 45 p.

Dalzell P., Adams T. and N. Polunin, 1996. Coastal fisheries in the Pacific Islands, Oceanography and Marine Biology : an annual review 34, pp. 395-531

Dalzell P. and G.L. Preston, 1992. Deep reef slope fishery resources of the South Pacific, a summary and analysis of the dropline fishing survey generated by the activities of the SPC fisheries programme between 1974 and 1988, Nouméa, South Pacific Commission, Inshore Fisheries Research project, tech. doc. 2, $299 \mathrm{p}$.

DAvid G., 1985. La pêche villageoise à Vanuatu : recensement I - moyens de production et production globale, Port-Vila, orstom, Notes et doc. d'océanographie 12, $198 \mathrm{p}$.

_ 1987. La pêche villageoise au Vanuatu : recensement 2 - La consommation de produits halieutiques dans la population, Port-Vila, ORSTOM, Notes et doc. d'océanographie 15, $122 \mathrm{p}$.

_, 1988. Le marché des produits de la pêche au Vanuatu, Port-Vila, ORSTOM, Notes et doc. d'Océanographie $18,115 \mathrm{p}$.

_, 1991. Pêche villageoise et alimentation au Vanuatu, exploration d'un système, Brest, thèse de doctorat de géographie de la mer, Skol Veur Breizh Izel, 1051 p.

_, 1999. Du village à la construction de l'État: l'agrandissement de la territorialité dans le Pacifique, in La Nation et le Territoire, Le territoire, lien ou frontière? t. 2, Paris, l'Harmattan, Géographie et Culture, pp. 235-252.

_, 2003. Mondialisation et recompositions territoriales et identitaires en Océanie Insulaire, in Îles rêvées, territoires et identités en crise dans le Pacifique insulaire, Paris, Presses de l'université Paris-Sorbonne, pp. 141-177.

_, 2004. La gestion des risques comme base de la viabilité des sociétés de l'Océanie insulaire, in David G. (éd.), Espaces tropicaux et risques, du local au global, Orléans, Presses universitaires d'Orléans / IRD, coll. CEDETE, pp. 353-364. 
—, 2005. Spatialité et temporalité océanes, Recherches sur les îles et les rivages de l'Océanie et de la partie occidentale de l'océan Indien. Paris, Université Paris IV-Sorbonne, Mémoire d'habilitation à diriger des recherches. Vol 1. Itinéraire d'un chercheur géographe de la mer et des îles, 369 p. multigr., vol. 2 Îles et rivages de l'Indo-Pacifique, variations géographiques, 428 p. multigr.

Désurmont A. et L. Chapman, 2000. The use of anchored FADs in the area served by the Secretariat of the Pacific Community (SPC) : regional synthesis, in Le Gall J.Y., Cayre P. et M. Taquet (éds), Pêche thonière et dispositifs de concentration de poissons, Paris, IFREMER, pp. 108-140.

Doumenge F.,1983. Aspects de la viabilité des petits États insulaires, étude descriptive. Genève, CNUCED, $39 \mathrm{p}$.

Dupouy C., Petit, M. et Y. Dandonneau, i 988. Satellite detected cyanobacteria bloom in the southwestern Pacific. Implication for oceanographic nitrogen fixation? International Journal of Remote Sensing 9, pp. 389-396.

EChInARd M., 1972. La pêche dans le lagon en Polynésie par le système des parcs à poissons, Journal de la Société des Océanistes 37, pp. 345-363.

FISHER, R.J, 1995. Collaborative management of resources for conservation and development, issues in forest conservation, Gland (Suisse), IUCN, 65 p.

Gilly B., 1989. Les modèles bioéconomiques en halieutique : démarches et limites, Cahiers de Sciences humaines 25 (1-2), pp. 23-33.

Gilmour D., 1990. Resource availability and indigenous forest management systems, Society and natural resources 3, pp. 145-158

Gordon H.S., 1954. The economic theory of common property resource: the fishery, Journal of Political Economy 62, pp. 124-142.

Grandperrin R., Gillet R., De Riviers X. et M. Theriault, 1982. Appâts vivants à Vanuatu: essais divers et récapitulatif, Port-Vila, Mission orstom, Notes et doc. d'océanogr. 2, 22 p.

Gulland J.A., 1983. Fish stock assessment, a manual of basic methods, New York, John Wiley \& Sons, FAO/Wiley Series on Food and Agriculture, 223 p.

Guilcher A., 1979. Précis d'hydrologie marine et continentale, Paris, Masson, $344 \mathrm{p}$.

Hviding E., 1989. Keeping the sea: aspects of marine tenure in Marovo lagoon, Solomon islands, in Ruddle K. and R.E. Johannes (eds.), Traditional marine resource management in the Pacific basin : an anthology, Jakarta, UNESCO/ROSTEA, pp. 7-44.

JoHANnes R.E, 1978. Traditional marine conservation methods in Oeania and their demise, Annual Review of Ecology and Systematics 9, 349-364.

-, 1981. Words of the Lagoon, fishing and marine lore in the Palau district of Micronesia. Berkeley, University of California Press, 245 p.
_, 1994. Pacific island peoples' science and marine management, in Science of Pacific island peoples, Suva, The University of the South Pacific, vol. 1, pp. 81-89.

—, 1998. The case for data-less marine resource management: examples from tropical nearshore fin fisheries, Trends in Ecology \&Evolution 13 (6), pp. 243246.

Johannes R.E, Green A. et T. AdAms, 2002. Les récifs coralliens du Pacifique : ressources et gestion, bilan et surveillance, Les récifs coralliens du Pacifique: état et suivi, ressources et gestion, Nouméa, IRD, Documents scientifiques et techniques II5, pp. 6986.

KULbiCKi M., I992. Present knowledge of the structure of coral reef fish assemblages in the Pacific, in Coastal resources and systems of the Pacific basin: investigation and steps toward protective management, Nairobi, UNEP regional Seas Reports and Studies 147, pp. 31-53.

Laurec A. et J.C. Le Guen, 1981. Dynamique des populations marines exploitées : concepts et modèles, Brest, CNEXO, Rapports scientifiques et techniques $45,118 \mathrm{p}$.

Laurent D., Bourdy G., Amade P., Cabalion P. et D. BourRet, 1993. La gratte ou ciguatera, ses remèdes traditionnels dans le Pacifique Sud, Paris, orsTOM, coll. Didactiques, $151 \mathrm{p}$.

Meyers R.F., 1989. Micronesian Reef Fishes. Guam, $298 \mathrm{p}$.

Moberg F. et P. RöNnBÄck, 2003. Ecosystem services of the tropical seascape: interactions, substitutions and restoration, Ocean and Coastal management 46, pp. 27-46.

Moffit R.B., 1993. Deepwater demersal Fish, in Right A and L. Hill, Nearshore Marine resources of the South Pacific, Suva, Institute of Pacific studies, chap. 4, pp. 73-95.

Nurse M. and M. Kabamba, 2000. Defining institutions for collaborative mangrove management: a case study from Tanga, Tanzania, Nairobi, IUCN, 16 p.

Pauly D., I997. Les pêches globales : géostratégie et émergence de nouveaux acteurs, Activités halieutiques et développement durable, $4^{\mathrm{e}}$ rencontres halieutiques de Rennes, 14-15 mars 1997, Rennes, ENSAR, p. 35-44.

Polovina J.J. and S. Ralston (eds.), 1987. Tropical snappers and groupers: biology and fisheries management, Boulder, Wetsview Press, 659 p.

Polunin N., 1989. Do traditional marine "reserves" conserve? a view of the Indonesian and New guinean evidence, in Ruddle K. and R.E. Johannes (eds.), Traditional marine resource management in the Pacific basin: an anthology, Jakarta, UNESCO/ROSTEA, pp. 191-211.

REVERET J.P., 1991. La pratique des pêches, comment gérer une ressource renouvelable, Paris, L'Harmattan, coll. Environnement, 198 p. 
Rougerie F. et B. WAUThy, 1986. Le concept d'endoupwelling dans le fonctionnement des atolls-oasis, Oceanologica Acta 9, pp. 133-148.

RUDDLE K.,1988. Social principles underlying traditional inshore fisheries management systems in the Pacific Basin, Marine Resources Economics 5 (4), pp. 351-363.

-, 1989. Traditionnal sole property rights and modern inshore fisheries management in the Pacific basin, in Campbell H., Menz, K. and G. Waugh (eds.), Economics of fishery management in the Pacific Islands region, Aciar proceedings 26, pp. 68-76.

Ruddle K. and T. AKichimi (eds.), 1984. Marine institutions in the Western Pacific, Osaka, National Museum of ethnology, Senri Ethnological studies, 17.

RudDle K. and R.E. Johannes (eds.), 1989. Traditional marine resource management in the Pacific basin : an anthology, Jakarta, UNESCO, 410 p.

SCHAEFFER M.B., 1954. Some aspects of the dynamics of populations important to the management of the commercial marine fisheries, Inter-Am. Trop. Tuna Comm. Bull. 1, pp. 25-56.

SEN S. et J.R. NiElSEN, 1996. Fisheries co-management: a comparative analysis, Marine Policy 20 (5), pp. 405-418.

Sims N.A., I989. Adapting traditional marine tenure and management practices to the modern fisheries framework in the Cook islands, in Ruddle K. and r.e. Johannes (eds), Traditional marine resource management in the Pacific basin: an anthology. Jakarta, UNESCO/ROSTEA, pp. 323-358.

South R., Goulet D., Tuqiri S. and M. Church (eds.), 1994. Traditional marine tenure and sustainable management of marine resources in Asia and the Pacific, Fiji, University of the South Pacific, 312 p.

SPC, 1993. South Pacific Economies Statistical Summary. Noumea, South Pacific Commission 13, 47 p.

TAYlor F.G.R., 1985. Distribution of the dinoflagellate Gambierdiscus toxicus in the eastern caribbean, Tahiti, Proceedings of the fifth international coral reef congress 4, pp. 423-428.

Thouret J.C. et F. LeONe, 2003. Aléas, vulnérabilités et gestion des risques naturels, in Moriniaux $\mathrm{V}$. (éd.), Les risques, Paris, Éditions du temps, coll. Questions de géographie, pp. 37-70.

Walmsley S.F., Howard C.H. and Medley P. 2005. Participatory fisheries stock assessment guidelines, London, MRAG, $166 \mathrm{p}$.

Williams D., 1990. Shallow-water reef fishes, in Vanuatu marine resources, Townsville, Australian Institute of Marine Science, pp. 66-76.

ZANN L.P., 1989. Traditional management and conservation of fisheries in Kiribati and Tuvalu atolls, in Ruddle K. and R.E. Johannes (eds.), Traditional marine resource management in the Pacific basin: an anthology, Jakarta, UNESCO/ROSTEA, pp. 77-101. 
